\title{
EVALUACIÓN DE Si APLICADO AL SUELO EN EL CRECIMIENTO, ABSORCIÓN Y SEVERIDAD DE ENFERMEDADES EN VIVERO DE PALMA ACEITERA
}

\author{
Gabriel Garbanzo ${ }^{1 / *}$, Eloy Molina $^{*}$, Gilberto Cabalceta $^{*}$, Edgardo Serrano $^{* *}$, Floria Ramírez,** \\ Palabras clave: Silicio; nutrición de plantas; fertilización; Inceptisol; Colletotrichum; vivero palma aceitera. \\ Keywords: Silicon; plant nutrition; fertilization; Inceptisol; Colletotrichum; oil palm nursery. \\ Recibido: 02/03/17 \\ Aceptado: 06/06/17
}

RESUMEN

Se evaluó el efecto de diferentes fuentes de $\mathrm{Si}$ en la severidad de enfermedades foliares (CNF) en plantas de vivero de palma aceitera (Elaeis guinensis) en el Pacífico Sur de Costa Rica. Las fuentes de silicio consistieron en la aplicación de $30 \mathrm{~g}$ de $\mathrm{SiO}_{2}$ por bolsa de 20,4 L de 2 fuentes de serpentinas (Simag y SMg), una fuente de diatomitas $(\mathrm{SiF})$, una fuente soluble (SKH) y 177 g por bolsa de $\mathrm{SiO}_{2}$ de un feldespato (Llane) mezclado en un Inceptisol éutrico. El diseño experimental fue de bloques completos al azar con 6 tratamientos y 4 repeticiones. Se evaluó a los 85, 127, 176, 219, 261, y 304 dds el crecimiento de las plantas y \% de severidad de CNF. Además, se realizaron análisis químicos de suelos y foliares en el tiempo, se evaluó el peso seco de plantas y se calculó la absorción de nutrimentos. Se encontró que el tratamiento SiF mostró mayor tolerancia a CNF significativamente $(\alpha: 0,05)$ en comparación al testigo, mientras que la fuente Simag mostró un mayor crecimiento. El Si presentó mayor absorción en Simag, test, $\mathrm{SMg}, \mathrm{SiF}$ y fueron significativas diferentes con respecto al SKH y Llane. El SKH en suelo fue el único tratamiento que aumentó los niveles de

\footnotetext{
1 Autor para correspondencia: Correo electrónico: juan.garbanzo@ucr.ac.cr

* Universidad de Costa Rica, Centro de Investigaciones Agronómicas, San José, Costa Rica.
}

\begin{abstract}
Si evaluation applied to soil in the growth, nutrient uptake and diseases severity in oil palm nursery. Si applied to soil in growth, nutrient uptake and diseases severity in oil palm nurseries was evaluated in the South Pacific of Costa Rica. The experiment consisted in the application of $30 \mathrm{~g} \mathrm{SiO}_{2}$ of 2 serpentines (Simag and $\mathrm{SMg}$ ), diatomaceous earth $(\mathrm{SiF})$, soluble silicon (SKH) and $177 \mathrm{~g} \mathrm{SiO}_{2}$ of feldspar (Llane) mixted with Eutric Inceptisol. The experiment design was randomized complete block with 6 treatments and 4 repetitions. The variables were evaluated at 85, 127, 176, 229, 261 and 304 days after planting (dds), and the percent of severity disease. Moreover, chemical soil and chemical foliar analysis were performed. The dry weight of plants was measured and the nutrient uptake was calculated. We found that the $\mathrm{SiF}$ treatment increased the tolerance toward CNF significantly, while the Simag increased plant growth. The highest accumulation of $\mathrm{Si}$ was presented mainly in Simag, test, $\mathrm{SMg}, \mathrm{SiF}$ treatment and differs significantly $(\alpha: 0,05)$ regarding SKH and Llane treatment. SKH was the only treatment that increased above

\footnotetext{
** Colono Agropecuario. Guápiles, Limón, Costa Rica.

*** Palma Tica S.A. Departamento de Investigación y Desarrollo, Corredores, Puntarenas, Costa Rica.
} 
Si por arriba de los $200 \mathrm{mg} . \mathrm{l}^{-1}$. A los $300 \mathrm{dds}$, el análisis de componentes principales correlacionó la severidad con las concentraciones de $\mathrm{Ca}$ en el suelo y mostró un comportamiento inverso a los contenidos de $\mathrm{Zn}, \mathrm{K}, \mathrm{P}, \mathrm{Mn}$ y en el tiempo. Se concluye que el $\mathrm{Si}$ en este suelo no presentó un efecto directo sobre el control de CNF, pero la fuente de diatomitas $(\mathrm{SiF})$ mostró mayor tolerancia, debido a que la enmienda facilita la absorción de micronutrimentos (Zn) al mejorar la nutrición de la planta. Asimismo, se encontró que la fuente $\mathrm{SKH}$ logró aumentar las concentraciones de $\mathrm{Si}$ en el suelo.

\section{INTRODUCCIÓN}

El Si es uno de los nutrimentos absorbidos en mayor cantidad por muchas plantas (Quero 2008). El Si constituye entre $0,1 \%$ y $10 \%$ del peso seco de las plantas superiores, se encuentra en mayor proporción en plantas monocotiledóneas que las dicotiledóneas (Epstein 1999, Aguirre et al. 2007). Los análisis químicos de tejidos en una amplia variedad de plantas determinaron concentraciones de Si entre 0,1 y 10\% (Quero 2008) y puede llegar a concentraciones superiores a $\mathrm{Ca}$, Mg, P, y K (Epstein 1999 y Ahmed et al. 2012).

Aunque el Si no se considera esencial para el crecimiento de las plantas, se han encontrado efectos benéficos en la aplicación en los cultivos, debido a un aumento en la resistencia de las plantas principalmente a estrés biótico y abiótico, que incluye la tolerancia a enfermedades (Epstein 1999, Ahmed et al. 2012, Raya y Aguirre 2012). Otras investigaciones demostraron incrementos en el crecimiento de plantas y mineralización eficiente de nutrimentos del suelo, cuando fueron sometidas a dosis de ácido monosilícico en la siembra de cultivos (Borda et al. 2007).

En las células epidermales se impregnan en la cutícula una doble capa de sílice $(2,5 \mu \mathrm{m})$ $\mathrm{y}$ al asociarse con las pectinas y polifenoles en
$200 \mathrm{mg} \cdot \mathrm{l}^{-1}$ the $\mathrm{Si}$ concentration in the soil. In $300 \mathrm{dds}$, the principal component analysis correlated the CNF with the Ca concentration in the soil, and presented an inverse behavior to the concentrations of $\mathrm{Zn}, \mathrm{K}, \mathrm{P}, \mathrm{Mn}$ and positively correlated with time. We concluded that $\mathrm{Si}$ does not control the CNF, but the diatomaceous earth $(\mathrm{SiF})$ increased the tolerance of $\mathrm{CNF}$, because the plant uptakes micronutrients (Zn) from the soil easily. In addition, the SKH source increased the $\mathrm{Si}$ concentration in the soil.

la pared celular, pueden formar barreras activas con la pérdida de agua, transpiración cuticular e infecciones fungosas (Ma y Yamaji 2008, Islam y Saha 1969, Datnoff et al. 2007 citado por Lobo 2013, Epstein 1999, Marschner y Marschner 2012). Las plantas mueven las formas de $\mathrm{H}_{4} \mathrm{SiO}_{4}$ a los trícomas de manera que surjan recubrimientos de las paredes externas de las células, una vez efectuado este proceso pueden proteger y proporcionar fortaleza mecánica a las estructuras celulares, principalmente de factores bióticos y abióticos (Quero 2008).

El aumento de la resistencia en la pared celular por acumulaciones de $\mathrm{Si}$ previenen el ingreso de haustorios de hongos fitopatógenos en las células (Ma y Yamaji 2008). Al estar fortalecida la pared celular, la segregación de enzimas liberadas por los hongos tampoco dañan la pared, lo cual evita el ingreso del patógeno y dificulta el proceso de infección en la planta (Arauz 2011). Los posibles mecanismos de defensa encontrados pertenecen a un proceso de inmovilización, que entre ellos incluyen la acumulación de lignina, compuestos fenólicos, quitinasas y peroxidasas (Epstein 1999 y Anderson 2012).

Investigaciones con fertilizantes silicatados $\left(\mathrm{SiO}_{2}\right)$, en la reducción de mal de Panamá 
(Fusarium oxysporum f. sp.) en cultivo de banano, encontraron una disminución en la severidad de los síntomas en las plantas, lo cual promueve una mayor biomasa en los tratamientos fertilizados con Si (Anderson 2012). Asimismo, trabajos realizados en palma aceitera demostraron reducción de enfermedades en pruebas exploratorias en Quepos, Costa Rica. Con aplicaciones de Si de 1,7 kg.planta ${ }^{-1}$ en distintos lotes, se encontraron tendencias a reducción de flecha seca (Acosta et al. 2007). Por otro lado, investigaciones en Guatemala evaluaron la aplicación de 150 a 250 g de ácido silícico al suelo, donde no encontraron diferencias estadísticas en las variables de crecimiento, pero se encontró una mayor acumulación de $\mathrm{P}$ en las hojas a dosis de $150 \mathrm{~g}$ de Si (Regil 2014). Sin embargo, no evaluaron presencia de enfermedades en la investigación.

En Costa Rica, los viveros de palma aceitera han demostrado problemas en el control de enfermedades, principalmente en la zona de Corredores, donde la precipitaciones se encuentran entre 4000 - 6000 mm.año ${ }^{-1}$. Entre las principales enfermedades encontradas en los viveros de palma aceitera se diagnosticó la antracnosis (Colletotrichum gloeosporioides) (Ortiz y Fernández 2000, ASD 2010). Sin embargo, los agentes causales encontrados en vivero pueden manifestarse según el crecimiento de la planta, condiciones climatológicas y manejo (Turner y Bull (1967), Renard y Quillec (1979), Turner (1981) citados en Ulloa y Serrano (2012)). Asimismo, mencionaron la presencia de otros agentes causales que provocan lesiones importantes en las láminas foliares, entre estos se encuentran: Botryodiplodia sp., Melanconium sp., Colletotrichum sp. [Glomerella sp.] y Curvularia sp. Según el porcentaje de severidad en las láminas foliares, pueden reducir el crecimiento de las plantas significativamente (Ramírez y Muñoz 2011, Ulloa y Ramírez 2011). En los viveros de palma aceitera en Costa Rica al ser producto de varios agentes patológicos que afecta a las láminas foliares, se le denominó localmente Complejo de Necrosis Foliar (CNF).
El CNF es un problema actual en vivero de palma aceitera, por lo que es necesario buscar nuevas alternativas para su manejo. Esta investigación tiene el objetivo de evaluar el efecto de fuentes de $\mathrm{Si}$ aplicadas al suelo en el crecimiento, absorción de nutrimentos y en la severidad de enfermedades foliares en viveros de palma aceitera.

\section{MATERIALES Y MÉTODOS}

La investigación se llevó cabo en la zona de vivero de la Compañía Palma Tica S.A., ubicada en Coto 47 en el cantón de Corredores en Puntarenas, Costa Rica. Esta zona se encuentra en la llanura aluvial de Coto-Corredores, con una elevación aproximada entre $24-30 \mathrm{msnm}$. La precipitación promedio anual registrada varía entre $3800-4500 \mathrm{~mm}$. Se procedió a medir las condiciones climatológicas durante el periodo de evaluación que comprendió desde julio de 2014 hasta marzo 2015, se registró una precipitación total $2051 \mathrm{~mm}$. La temperatura promedio durante el periodo fue max: $36,4^{\circ} \mathrm{C}$ y min: 26,5 , con una humedad relativa promedio de $87,4 \%$ y una radiación solar promedio de $377,2 \mathrm{~W} \mathrm{~m}^{-2}$.

Se ubicaron "boquetes" dentro del vivero donde las plantas estuvieran en una zona con historial de problemas de enfermedades foliares. Estos se seleccionaron con las especificaciones de: fácil acceso, con un área de $900 \mathrm{~m}^{2}$, de manera que se acomodaran 30 plantas de fondo por 24 filas. Se trasladó suelo aluvial (Inceptisol) utilizado en el periodo 2014 para el llenado de las bolsas de vivero en Coto $47 \mathrm{y}$, posteriormente, se cubrió con un plástico transparente para reducir pérdidas por lixiviación y escorrentía.

Se seleccionaron plantas de E. guineensis del material Compacta x Ghana. Estas fueron sembradas en pre-vivero con Jiffi y fueron manejadas durante 2 meses bajo condiciones controladas. La nutrición de estas plántulas se manejó bajo fuentes de lenta liberación (Osmocote $\left.{ }^{\circledR}\right)$ y aplicaciones foliares en conjunto con fungicidas preventivos. Las plantas se seleccionaron de manera que el material fuera totalmente homogéneo y se desecharon plantas elongadas, con presencia de 
enfermedades foliares, mal desarrolladas, entre otros. Posteriormente, se adquirieron bolsas de 25,4 $\mathrm{cm}$ de diámetro y $40 \mathrm{~cm}$ de altura ( $\mathrm{vol}=20,4 \mathrm{~L})$, estas bolsas se utilizaron para la etapa de vivero.

Se procedió a buscar fuentes de Si presentes en Costa Rica que fueran similares a un producto $\mathrm{SiO}_{2}$ feldespatico, debido a que es la fuente utilizada comúnmente en los viveros de Palma Tica. Al observar que la curva de absorción en la planta requiere de al menos 25 gramos de macroelementos (Ramírez y Muñoz 2010), se estimó una cantidad de 30 gramos de $\mathrm{SiO}_{2}$ en la planta, con el fin de calcular la cantidad del elemento requerida por la fuente para el suministro comparativo de $\mathrm{Si}$ entre los tratamientos. Después, se seleccionaron 3 fuentes de Si granular y una líquida. Se calcularon las dosis correspondientes para el volumen de suelo que contenían las bolsas de vivero y se pesó en balanza digital para posteriormente ser almacenadas en bolsas plásticas transparentes de polietileno de 11 x $20 \mathrm{~cm}$.
Las fuentes de Si fueron mezcladas con el suelo de forma manual (Cuadro 1). Se cuantificó el número de palas equivalente a una bolsa de 20 L según el suelo húmedo y seco. Para realizar las mezclas se utilizó un manteado de capa gruesa para evitar pérdidas. Se usó el número de palas correspondientes al volumen de suelo que requieren una bolsa de vivero y se mezcló las dosis de las fuentes de Si para cada tratamiento (Cuadro 2). Luego se llenó la bolsa completamente y se golpeó contra el suelo para evitar las cavidades de aire, asimismo, se alinearon entre los boquetes, se etiquetaron (enumeradas) cada una de las bolsas y se dejaron por 30 días en campo. El tratamiento testigo y SKH se dejaron con suelo solamente, para luego aplicar el tratamiento SKH después de las siembras de las palmas. Es importante mencionar que se recolectaron muestras para un análisis químico de suelo antes de realizar las mezclas con las fuentes de Si (Cuadro 3).

Cuadro 1. Características químicas de fuentes de Si utilizadas en el ensayo de vivero de E. guineensis Coto. Costa Rica.

\begin{tabular}{|c|c|c|c|c|c|c|c|c|}
\hline \multirow{3}{*}{ Productos } & \multicolumn{7}{|c|}{$\% \mathrm{p} / \mathrm{v}$} & \multirow{3}{*}{ Otros } \\
\hline & \multirow{2}{*}{$\begin{array}{l}\text { Total } \\
\mathrm{SiO}_{2}{ }^{*}\end{array}$} & \multicolumn{2}{|c|}{ Soluble ${ }^{+}$} & \multirow{2}{*}{$\mathrm{K}_{2} \mathrm{O}$} & \multirow{2}{*}{$\mathrm{CaO}$} & \multirow{2}{*}{$\mathrm{MgO}$} & \multirow{2}{*}{ S } & \\
\hline & & $\mathrm{SiO}_{2}$ & $\mathrm{Si}$ & & & & & \\
\hline Llanero & 59 & 0,81 & 0,38 & 0,27 & 10,7 & 0,27 & 3,69 & - \\
\hline SILIMAG & 30 & 0,32 & 0,15 & - & 3,72 & 32,9 & 1,94 & - \\
\hline Stan SKH & 20 & - & - & 18 & - & - & - & Aci. húmicos \\
\hline Sulfato de magnesio & 20 & 3,91 & 1,83 & - & 2,84 & 17,9 & 10,2 & - \\
\hline Silycal-Flow & 70 & 0,64 & 0,30 & 0,17 & 17,1 & 0,64 & 0,44 & - \\
\hline
\end{tabular}

Análisis realizados en Laboratorio de Suelos y Foliares Compañía palma Tica S.A.

+Metodología empleada según Sebastian et al. (2013) para fertilizantes solidos con Si.

*Silicio total reportada por fabricante de producto. 
Cuadro 2. Dosis de fuentes de Si correspondientes a 30 gramos de Si elemental para aplicación en bolsas de 20 L para vivero de E. guineensis en Coto. Costa Rica.

\begin{tabular}{|c|c|c|c|c|c|}
\hline & Tratamiento & Signo & Dosis & $\begin{array}{l}\mathrm{SiO}_{2} \\
(\mathrm{~g} . \text { bolsa } \\
\end{array}$ & Observaciones \\
\hline 1 & Testigo & test & --- & 0 & Fórmula usada en viveros \\
\hline 2 & Llanero & Llane & 300 g.bolsa ${ }^{-1}$ & 177 & - \\
\hline 3 & SILIMAG & Simag & 100 g.bolsa ${ }^{-1}$ & 30 & - \\
\hline 4 & Stan SKH* & SKH & $150 \mathrm{ml} \cdot$ bolsa $^{-1}$ & 30 & 400 1.ha $^{-1}$ drench \\
\hline 5 & Sulfato de magnesio & $\mathrm{SMg}$ & 150 g.bolsa ${ }^{-1}$ & 30 & - \\
\hline 6 & Silycal-Flow & $\mathrm{SiF}$ & 43 g.bolsa ${ }^{-1}$ & 30 & - \\
\hline
\end{tabular}

*5 aplicaciones de 30 ml.planta ${ }^{-1}$

Cuadro 3. Análisis químico de suelo inicial.

\begin{tabular}{|c|c|c|c|}
\hline Mehlich-3 & & $\mathrm{NC}$ & Inicial \\
\hline pH $\mathrm{H}_{2} \mathrm{O}$ (agua) & \multirow{6}{*}{ 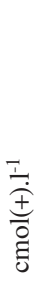 } & 5,5 & 6,42 \\
\hline Acidez (KCl) & & 0,5 & 0,30 \\
\hline $\mathrm{Ca}$ & & 4 & 34,5 \\
\hline $\mathrm{Mg}$ & & 1,1 & 3,8 \\
\hline K & & 0,2 & 0,55 \\
\hline CICE & & 5 & 39,1 \\
\hline SA & \multirow[t]{5}{*}{$\%$} & - & 0,77 \\
\hline $\mathrm{P}$ & & 15 & 22,4 \\
\hline $\mathrm{Zn}$ & & 1,7 & 2,64 \\
\hline $\mathrm{Cu}$ & & 0,4 & 19,5 \\
\hline $\mathrm{Fe}$ & & 10 & 308 \\
\hline $\mathrm{Mn}$ & \multirow{2}{*}{$\stackrel{I}{\dot{0}}$} & 4 & 36 \\
\hline $\mathrm{Si}^{*}$ & & 200 & 170 \\
\hline
\end{tabular}

+ Solución extractora Mehlich 3, Laboratorio Suelos y Foliares Palma Tica S.A.; $\mathrm{NC}=$ nivel crítico para Inceptisoles según Cabalceta y Molina (2006). CICE = Capacidad de intercambio de Cationes Efectiva = Acidez $+\mathrm{Ca}+\mathrm{Mg}+\mathrm{K}$. *. Metodología de extracción con ácido acético según Lobo (2013).

Previo a la siembra de las plantas, se realizó una aplicación de herbicida en toda el área de vivero, con el fin de realizar un manejo total de las malezas. Dos semanas después se trasplantaron plántulas de E. guineensis. El tratamiento con la fuente de Si líquido (Stan SKH) se aplicó en forma de "drench" dirigido al suelo en las bolsas.
Se usó un volumen de 500 1.ha ${ }^{-1}$ de caldo, lo que equivale a $30 \mathrm{ml}$ de SKH y $30 \mathrm{ml}$ de agua. Este se realizó en forma escalonada a los 3, 5, 7, 9 y 10 meses después de siembra por tratarse de una fuente líquida con el fin de reducir la pérdida por lavado y lixiviación, producto de las altas precipitaciones en la zona. 
Se realizó el mismo manejo agronómico utilizado por el departamento de viveros, con excepción de la nutrición y el combate de enfermedades foliares. La aplicación de fertilizante se llevó a cabo de acuerdo con el fraccionamiento propuesto por la unidad de investigación. Los nutrimentos se aplicaron en forma granular en el área superficial de la bolsa, distanciados del bulbo para no producir pérdida de agua en la planta por difusión.

La fertilización se llevó a cabo con base en la curva de absorción propuesta por Ramírez y Muñoz (2011), donde se midió el consumo total de nutrimentos en las plantas de vivero correspondiente a 365 días antes de ser sembradas en sitios definitivos (Cuadro 4). Posteriormente, en el 2012 se realizó una validación de la curva, donde se evaluaron distintas dosis de nutrimentos, incluyendo las aplicaciones convencionales en vivero con base en la absorción de las plantas, lo cual demostró su efectividad y su validez en el crecimiento entre las distintas aplicaciones. La fórmula que se utilizó en vivero propuesto por Palma Tica corresponde a 15,50 $9,76-15,50-4,00-3,20(\mathrm{~S})-0,30(\mathrm{~B})$, constituidas por las fuentes de nitrato de amonio (NAM), microessentials (SZ), cloruro de potasio $(\mathrm{KCl})$, kiessierita y granubor.

Cuadro 4. Fertilización propuesta con base en la curva de absorción en plantas de E. guinensis en etapa de vivero.

\begin{tabular}{|c|c|c|c|c|c|c|c|}
\hline Consumo curva & $\mathrm{N}$ & $\mathrm{P}$ & K & $\mathrm{Mg}$ & S & $\mathrm{Zn}$ & B \\
\hline Eficiencia*/dds** & $60 \%$ & $50 \%$ & $70 \%$ & $80 \%$ & $50 \%$ & $50 \%$ & $10 \%$ \\
\hline \multicolumn{8}{|c|}{ - } \\
\hline 82 & 0,05 & 0,01 & 0,03 & 0,02 & 0,01 & 0,15 & 0,63 \\
\hline 149 & 0,93 & 0,09 & 0,26 & 0,07 & 0,06 & 1,01 & 2,03 \\
\hline 210 & 2,79 & 0,27 & 1,03 & 0,24 & 0,19 & 2,53 & 6,52 \\
\hline 268 & 5,94 & 0,72 & 2,98 & 0,56 & 0,51 & 8,29 & 25,02 \\
\hline 298 & 8,71 & 1,03 & 4,76 & 0,80 & 0,73 & 11,52 & 29,91 \\
\hline 335 & 17,48 & 2,25 & 10,28 & 1,69 & 1,64 & 29,13 & 61,77 \\
\hline 363 & 24,40 & 3,01 & 13,09 & 2,45 & 2,45 & 47,49 & 94,89 \\
\hline
\end{tabular}

* Eficiencia fertilización propuesta. ** días después de siembra

\section{Variables evaluadas en vivero}

Una vez establecidas las plantas en vivero se evaluaron a los $85,130,175,220,265$ y 300 dds, las variables se distribuyeron en: crecimiento morfológico de las plantas, evaluación de severidad de enfermedades foliares, análisis químico en plantas.

Entre las variables morfológicas se evaluaron: el largo de raquis, que consistió en medir la hoja 1 desde la parte proximal de pecíolo hasta el meristemo apical de la hoja. El diámetro de bulbo, que consistió en medir con un vernier el ancho del bulbo de la planta a una altura de $1-2$ cm desde la emisión de la raíz, además, se realizó un conteo de la cantidad de hojas totales completamente formadas a partir de la hoja 1 (Rodríguez y Jiménez 2013).

Las evaluaciones de severidad del CNF, se realizaron mediante una escala visual propuesta por la Compañía Palma Tica S.A. (Ulloa y Ramírez 2011), la escala fue representada por 5 grados 
que determinan el porcentaje de daño en la hoja 1 y planta total. Están constituidos de la siguiente manera: $1=$ representa el $3 \%$ de la hoja o planta dañada, $2=7 \%, 3=15 \%, 4=40 \%, 5=75 \%$.

El análisis de la concentración total de nutrimentos en las plantas se realizó mediante la selección de una planta promedio. Esta se efectuó mediante una selección estadística por medio de intervalos de confianza a partir de los datos morfológicos (diámetro de bulbo, largo de raquis y hojas totales). Los intervalos de confianza se calcularon al evaluar el tamaño de la muestra y la desviación estándar con un alfa $(\alpha)=0,01$.

Después, se calculó el límite superior y el límite inferior según la media de los datos. La selección de las plantas se estimó mediante un filtrado de las observaciones para cada repetición, donde se incluía los límites inferior y superior correspondiente para cada una de las variables morfológicas. De las repeticiones que mostraron más de 2 plantas con los intervalos de confianza estimados, se eligió la planta que mostró el diámetro de bulbo más cercano a la media de las observaciones.

Una vez identificadas las plantas para el análisis de absorción, se recolectó una planta por cada repetición en las parcelas experimentales, se lavó con abundante agua el suelo de las bolsas y se separaron la parte aérea y las raíces. Ambas partes se pesaron y se colocaron en hornos durante 3 días a $65^{\circ} \mathrm{C}$ y luego se evaluó el peso seco para luego incluir las muestras al laboratorio, esta metodología se desarrolló según Ramírez y Muñoz (2011). Los muestreos se realizaron a los 85, 127, 176, 219, 261, 304 días después de siembra.

El análisis foliar de las plántulas se realizó mediante la selección de la hoja 1 de acuerdo con la metodología de Rodríguez y Jiménez (2013). Esta consistió en evaluar 2 foliolos de cada lado para hojas normales y para hojas bifurcadas o lanceoladas se recolectó completamente la hoja. Este análisis comenzó a partir de los 164 dds, debido al tamaño de las plantas, ya que no se recolectaba el peso suficiente para el análisis de laboratorio, los demás muestreos se realizaron a los 220 - 265 - 300 dds. Posteriormente, las muestras fueron enviadas al Laboratorio de Suelos y Foliares de la Compañía Palma Tica S.A. y se realizaron determinaciones de Si mediante la metodología de Lobo (2013), N por combustión seca, $\mathrm{P}, \mathrm{K}, \mathrm{Ca}, \mathrm{Mg}, \mathrm{S}, \mathrm{Fe}, \mathrm{Zn}, \mathrm{Mn}, \mathrm{Cu}$, a través de la digestión húmeda con $\mathrm{HNO}_{3}$ y determinación por espectrometría de emisión atómica con plasma (ICP).

Los análisis químicos de los suelos en las bolsas se realizaron a los 90, 170, $260 \mathrm{dds}$. Las muestras se recolectaron a 2 profundidades de $0-15$ y 15 - 30 utilizando un submuestreo de manera sistemática en 15 bolsas por repetición alternadas entre plantas. Las muestras fueron enviadas al Laboratorio de Suelos y Foliares de la Compañía Palma Tica S.A., se determinaron $\mathrm{pH}$, acidez intercambiable, $\mathrm{Ca}, \mathrm{Mg}, \mathrm{K}$, $\mathrm{P}, \mathrm{Fe}, \mathrm{Zn}, \mathrm{Mn}, \mathrm{Cu}$ mediante extracciones con Mehlich III de acuerdo con la metodología de Cabalceta y Molina (2006), Al por retitulación con $\mathrm{HCl}$ al 0,01 M y Si según la metodología de Lobo (2013).

\section{Diseño experimental}

El experimento consistió de 6 tratamientos y 4 repeticiones con un diseño experimental de bloques completos al azar. Se montaron 4 bloques, cada bloque experimental fue representado por 240 plantas con un tamaño de $302 \mathrm{~m}^{2}$, las repeticiones fueron ordenadas en filas, cada repetición presentaba 30 plantas. Los bloques fueron ubicados en un sector plano que no mostraran problemas de topografía.

Para el análisis de los datos morfológicos se realizaron índices de áreas bajo la curva (abc) para reunir la información de las variables en el tiempo (Ecuación 1). Esta metodología consistió en calcular el área de un trapecio entre 2 fechas de evaluación, se tomó en cuenta las evaluaciones morfológicas en cada fecha y los días transcurridos para realizar una sumatoria 
de las áreas calculadas entre los periodos. La sumatoria de las áreas determinó el índice de abc, lo cual mide el efecto de los tratamientos en trascurso del tiempo.

$$
\sum \frac{b+B \times a}{2}
$$

\section{Ecuación 1}

Donde:

$\mathrm{a}=$ número de días trascurridos entre $\mathrm{t} 1$ y $\mathrm{t} 2$.

$\mathrm{b}=$ medida en el tiempo $1(\mathrm{t} 2)$.

$\mathrm{B}=$ medida en el tiempo 2 (t1).

Posteriormente, los datos fueron sometidos a un análisis de varianza, se calcularon las diferencias estadísticas mediante pruebas de Dunnett test $(\alpha=0,05)$ para los análisis morfológicos y LSD $(\alpha=0,05)$ para los análisis químicos. Por último, para explicar la variabilidad del sistema se procedió a realizar un análisis multivariado, mediante un análisis de componentes principales (CP) (Abdi y Williams 2010, Jolliffe 2005, Serrano 2003), con el fin de encontrar una posible estructura en la base de datos y explicar la variación según las dimensiones calculadas. Los cálculos fueron realizados, a través el programa estadístico Rstudio versión 0.98.1102.

\section{RESULTADOS}

\section{Efecto de las fuentes de $\mathrm{Si}$ en el crecimiento de las plantas y severidad de enfermedades foliares}

Las fuentes de $\mathrm{Si}$ mostraron incrementos en el crecimiento y tolerancia a CNF en vivero de palma aceitera (Cuadro 5). Al evaluar las variables morfológicas cada 45 días, el índice de área bajo la curva $(\mathrm{abc})$ en el diámetro de bulbo presentó diferencia significativa $(\alpha=0,01)$ en el Simag en comparación al test (testigo) según la prueba de Dunnett test ( $\mathrm{pr}>|\mathrm{t}| \mathrm{l})$. El abc en el largo de hoja y largo de raquis no mostraron incrementos importantes en relación con el testigo. No obstante, se encontraron diferencias estadísticas en el abc de hojas totales en Simag y $\mathrm{SiF}$ en comparación al testigo $(\mathrm{p}<0,028)$. En relación con la incidencia de las enfermedades foliares en vivero, las mismas estuvieron presentes en todas las evaluaciones y se encontró que el abc en severidad en la hoja 1 y total presentaron diferencias altamente significativas al comparar el tratamiento $\mathrm{SiF}$ con el testigo $(\mathrm{p}<0,001)$, el resto de los tratamientos no manifestaron reducciones en la severidad. 
Cuadro 5. Efecto de diferentes fuentes de Si en el índice de área bajo la curva en crecimientos morfológicos y severidad del CNF de plantas de palma aceitera.

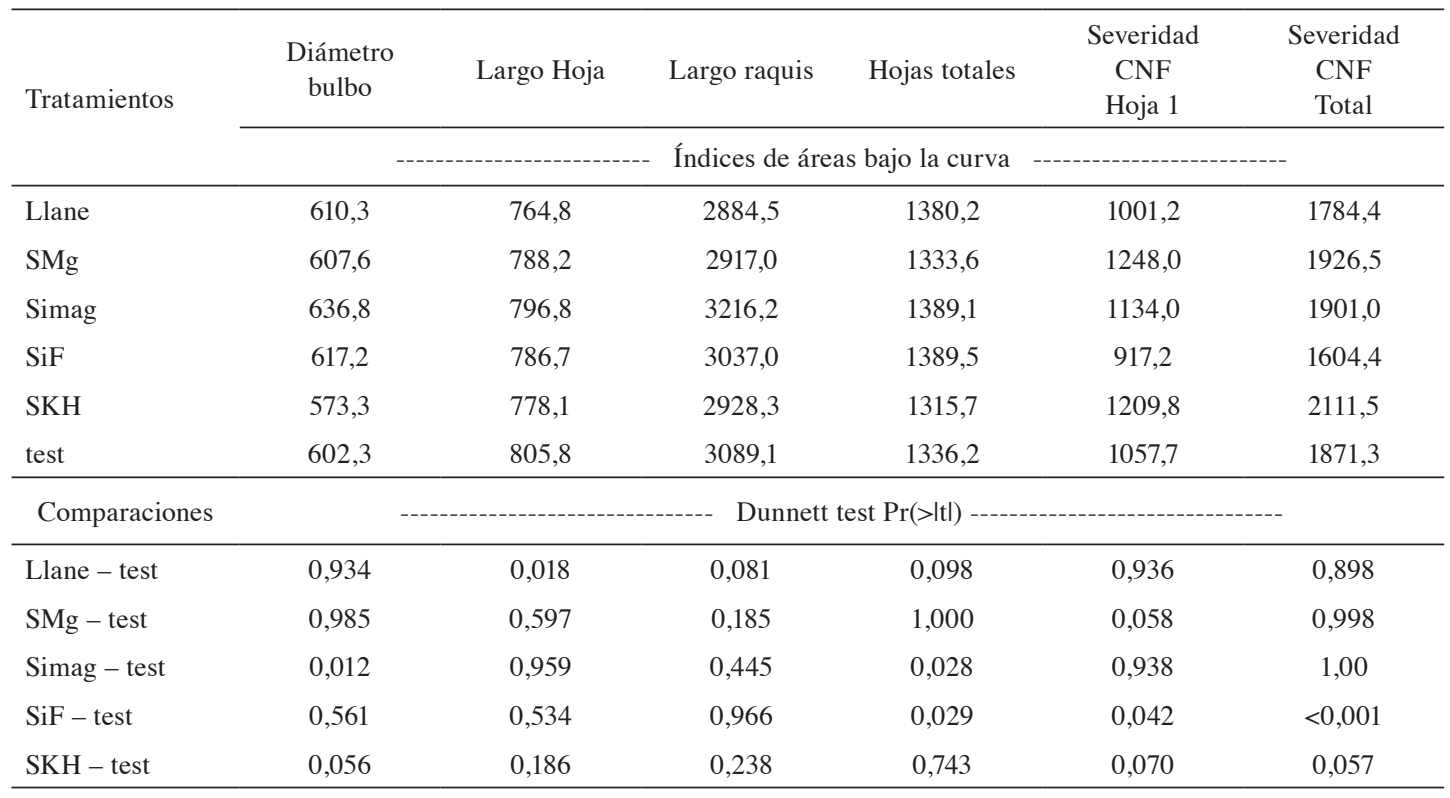

$\mathrm{n}=22525$; Variables morfológicas y severidad evaluados a los 85, 127, 176, 219, 261, 304 días después de siembra. Probabilidades por debajo de 0,05 (Pr>|t|) representan diferencias significativas de tratamientos en comparación con el testigo.

Se detectaron incrementos en biomasa y materia seca al aplicar fuentes de Si. En relación con el peso húmedo en la parte aérea y raíz, el abc presentó diferencias $(\alpha=0,01)$ en el Simag al compararlo con el testigo o referente (Cuadro 6), las demás pruebas no manifestaron el mismo efecto. Al evaluar el peso seco para ambas partes de las plantas, no mostró diferencias, sin embargo, se encontraron tendencias de mayor acumulación de materia seca en el Simag. Al realizar la sumatoria de los pesos secos de ambas partes de la planta sí se encontraron diferencias $(\alpha=0,01)$ al comparar el Simag con el testigo, los demás no exhibieron diferencias. 
Cuadro 6. Índice de área bajo la curva en peso húmedo, seco y seco total en plantas de palma aceitera tratadas con distintas fuentes de Si en vivero.

\begin{tabular}{|c|c|c|c|c|c|}
\hline \multirow[t]{2}{*}{ Tratamientos } & $\begin{array}{l}\text { Peso húmedo } \\
\text { aérea }\end{array}$ & $\begin{array}{c}\text { Peso húmedo } \\
\text { Raíz }\end{array}$ & Peso seco aéreo & Peso seco Raíz & Peso seco total \\
\hline & \multicolumn{5}{|c|}{ Índices de áreas bajo la curva } \\
\hline Llane & 26135,1 & 10014,7 & 6430,6 & 1475,5 & 7906,1 \\
\hline $\mathrm{SMg}$ & 28126,8 & 9864,6 & 7109,3 & 1463,2 & 8572,5 \\
\hline Simag & 34877,2 & 12444,2 & 8211,5 & 1805,1 & 10016,8 \\
\hline $\mathrm{SiF}$ & 28739,9 & 10808,6 & 6901,5 & 1538,5 & 8440,0 \\
\hline SKH & 25034,7 & 9790,4 & 6196,4 & 1415,2 & 7611,6 \\
\hline test & 27472,7 & 10630,4 & 7177,8 & 1570,3 & 8748,1 \\
\hline Comparaciones & \multicolumn{5}{|c|}{ 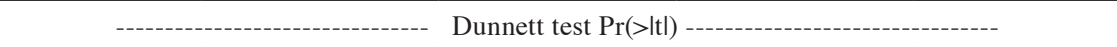 } \\
\hline Llane - test & 0,94 & 0,99 & 0,28 & 0,99 & 0,95 \\
\hline $\mathrm{SMg}$ - test & 1,00 & 1,00 & 0,99 & 0,99 & 0,20 \\
\hline Simag - test & 0,01 & 0,01 & 0,08 & 0,06 & $<0,01$ \\
\hline $\mathrm{SiF}$ - test & 0,96 & 0,54 & 0,94 & 0,86 & 0,31 \\
\hline SKH - test & 0,66 & 0,70 & 0,11 & 0,73 & 0,11 \\
\hline
\end{tabular}

$\mathrm{n}=144$; Variables de crecimiento evaluadas a los 85, 132, 180, 223, 265, 308 días después de siembra. Probabilidades por debajo de $0,05(\operatorname{Pr}>|t|)$ representan diferencias significativas de tratamientos en comparación al testigo.

Se identificaron tendencias a una mayor acumulación de materia seca y desarrollo de hojas totales en la mayoría de ensayos a los 300 dds cuando se compararon con el testigo (Cuadro 7). El diámetro de bulbo $(\mathrm{cm})$ y largo de raquis $(\mathrm{cm})$ no presentaron diferencias en los grupos evaluados $(\alpha=0,05)$ cuando se comparó con el testigo, no obstante, el Simag y $\mathrm{SiF}$ manifestaron tendencias de mayor crecimiento (5\%). El desarrollo de hojas totales mostró diferencias significativas $(\alpha<0,001)$ igualmente cuando el Simag se comparó, el resto de las pruebas no reveló diferencias. En la acumulación de materia seca se encontró un mayor peso seco (g) en el SMg y Simag con un 10 y $14 \%$ más de materia que el referente, respectivamente, el resto no mostraron diferencias con la particularidad que el SiF evidenció tendencias $(\mathrm{pr}=0,06)$. El porcentaje severidad en la hoja $1 \mathrm{y}$ severidad total (\%) en las plantas no manifestó diferencias entre las pruebas. 
Cuadro 7. Efecto de diferentes fuentes de Si en el crecimiento morfológico, peso seco total y severidad del CNF en plantas de palma aceitera a los $300 \mathrm{dds}$.

\begin{tabular}{|c|c|c|c|c|c|c|}
\hline Tratamientos & $\begin{array}{c}\text { Diámetro } \\
\text { bulbo }\end{array}$ & Largo raquis & Hojas totales & $\begin{array}{c}\text { Peso seco } \\
\text { Total }\end{array}$ & $\begin{array}{c}\text { Severidad } \\
\text { Hoja } 1\end{array}$ & $\begin{array}{c}\text { Severidad } \\
\text { Total }\end{array}$ \\
\hline & $\mathrm{cm}$ & $\mathrm{cm}$ & $\mathrm{u}$ & $\mathrm{g}$ & $\%$ & $\%$ \\
\hline Llane & 5,23 & 34,4 & 9,03 & 131,5 & $4,54 a^{*}$ & $10,23 \mathrm{a}$ \\
\hline $\mathrm{SMg}$ & 5,03 & 35,4 & 9,21 & 151,2 & $3,90 \mathrm{a}$ & $8,94 \mathrm{a}$ \\
\hline Simag & 5,41 & 39,1 & 9,47 & 144,7 & $4,54 \mathrm{a}$ & $9,51 \mathrm{a}$ \\
\hline $\mathrm{SiF}$ & 5,34 & 36,5 & 8,90 & 138,3 & $3,97 \mathrm{a}$ & $8,71 \mathrm{a}$ \\
\hline SKH & 4,86 & 35,8 & 8,99 & 109,8 & $3,72 \mathrm{a}$ & 9,99 a \\
\hline test & 5,19 & 37,4 & 8,81 & 130,8 & $3,92 \mathrm{a}$ & 8,86 a \\
\hline Comparaciones & \multicolumn{4}{|c|}{--- Dunnett test $\operatorname{Pr}(>|t|)$ - } & \multicolumn{2}{|c|}{ Kruskal Wallis } \\
\hline Llane - test & 0,997 & 0,027 & 0,581 & 0,197 & - & - \\
\hline $\mathrm{SMg}$ - test & 0,432 & 0,229 & 0,081 & 0,006 & - & - \\
\hline Simag - test & 0,141 & 0,567 & $<0,001$ & 0,020 & - & - \\
\hline $\mathrm{SiF}-$ test & 0,454 & 0,912 & 0,986 & 0,063 & - & - \\
\hline SKH - test & 0,012 & 0,491 & 0,742 & 0,220 & - & - \\
\hline
\end{tabular}

$\mathrm{n}=2988$; Variables de crecimiento evaluadas a los 308 días después de siembra; Probabilidades por debajo de 5\% (Pr (>lt) $<0,05)$ representan diferencias significativas de tratamientos en comparación al testigo según prueba de Dunnett. * Comparaciones múltiples según prueba de Krukal Wallis p.adj = Bonferroni. $\alpha=0,05$.

\section{Efectos de las fuentes de Si en la concentración de nutrimentos en el suelo}

El pH del suelo se redujo en el tiempo (Cuadro 8). El pH evaluado hasta una profundidad de $0-15 \mathrm{~cm}$ mostró reducción significativa $(\alpha=0,05)$ a los 175 dds y 265 dds en comparación a la concentración inicial. El SKH mantuvo el mismo $\mathrm{pH}$ en forma significativa en el tiempo, el resto de los tratamientos no presentaron diferencias mínimas a excepción de Simag, SiF, y SMg a los 175 dds. Al evaluar el pH a una profundidad de $15-30 \mathrm{~cm}$ no evidenció diferencias a los 85 dds y 175 dds entre los tratamientos, no obstante, a los 265 dds, el Llane y SMg mostraron diferencias importantes en comparación a los demás. Al comparar las 2 profundidades presentaron tendencias de un comportamiento creciente en el $\mathrm{pH}$ conforme aumenta la profundidad. 


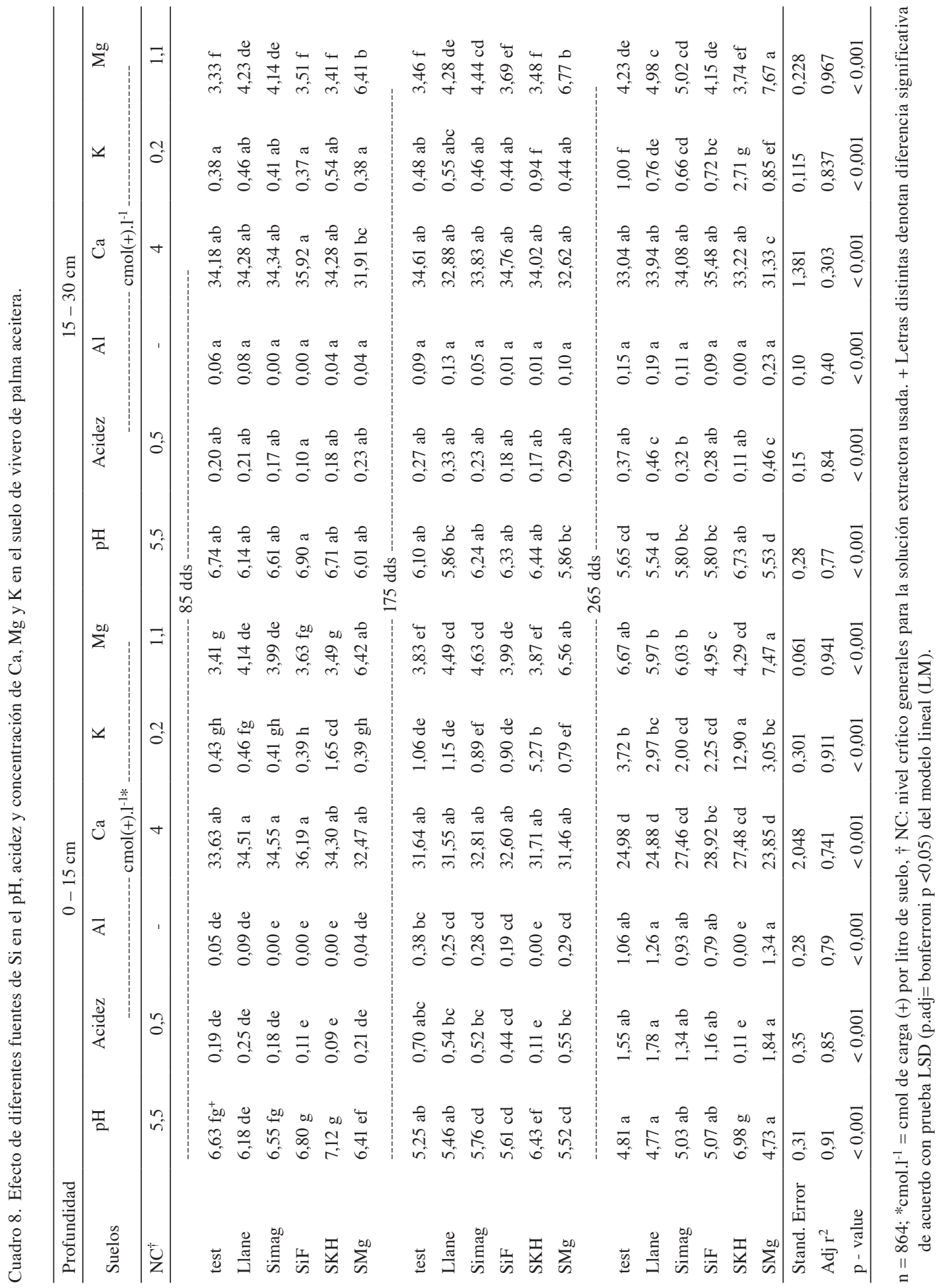


La acidez intercambiable a una profundidad de $0-15 \mathrm{~cm}$ presentó un comportamiento creciente entre los 175 y 265 dds, con diferencias entre tratamientos. El SKH mostró la menor concentración de acidez y fue estadísticamente diferente $(\alpha=0,05)$ del resto. Todas las evaluaciones presentaron aumentos de acidez intercambiable a los 265 dds con excepción del silicato de potasio SKH. El efecto de la acidez a profundidad de 15 - $30 \mathrm{~cm}$ no evidenció cambios hasta los 265 dds, donde el Llane y SMg presentaron la mayor acidez y difieren estadísticamente del resto de las pruebas. En relación con la concentración de Al a profundidad de $0-15 \mathrm{~cm}$ el SKH reveló diferencias en comparación al resto de los ensayos a los 175 y 265 dds. El comportamiento del Al a profundidad de $15-30 \mathrm{~cm}$ no presentó diferencias para todos los experimentos en las 3 fechas. Al evaluar ambas profundidades se encontró un comportamiento decreciente en la acidez y Al intercambiable cuando aumentó la profundidad.

Las concentraciones iniciales de $\mathrm{Ca}$ en el suelo superaron aproximadamente 8 veces el nivel crítico para inceptisoles. El Ca en los primeros $15 \mathrm{~cm}$ de los suelos no mostró diferencias significativas al comparar todos los tratamientos a los 85 y 175 dds, sin embargo, a los 265 dds hubo disminución del nivel de $\mathrm{Ca}$. En la profundidad de 15 - $30 \mathrm{~cm}$ no hubo diferencias en el contenido de $\mathrm{Ca}$. Por otro lado, las concentraciones de $\mathrm{K}$ aumentaron con el tiempo en todas los tratamientos en consecuencia del efecto residual del fertilizante aplicado. Esto fue 8 veces mayor $\left(1,65 \mathrm{cmol}(+) \cdot \mathrm{l}^{-1}\right)$ al nivel crítico $\left(0,2 \mathrm{cmol}(+) \cdot \mathrm{l}^{-1}\right)$. A los 175 y 265 dds el ensayo SKH presentó las concentraciones más altas de $\mathrm{K}$, con diferencias en comparación a las demás pruebas, lo mismo ocurrió en la profundidad de $15-30 \mathrm{~cm}$. Este efecto fue causado por la alta concentración de K que presentó el SKH y que aportó al suelo en el transcurso del experimento.
El contenido de $\mathrm{Mg}$ en el suelo mostró un incremento significativo con las fuentes de $\mathrm{Si}$ que suministraron $\mathrm{Mg}$. El $\mathrm{SMg}$ evidenció la mayor $\left(6,42 \mathrm{cmol}(+) \cdot 1^{-1}\right)$ de $0-15 \mathrm{~cm}$, con diferencias $(\alpha=0,05)$ en comparación al resto de las pruebas. Este comportamiento se mantuvo a los $175\left(6,56 \mathrm{cmol}(+) .1^{-1}\right)$ y $265 \mathrm{dds}(7,47$ cmol(+). $\left.1^{-1}\right)$ para el mismo manejo, el cual es comparado hasta la última fecha con el testigo $\left(6,67 \mathrm{cmol}(+) \cdot \mathrm{l}^{-1}\right)$. La concentración de $\mathrm{Mg}$ a profundidad de 15 - $30 \mathrm{~cm}$ mostró el mismo incremento en el tiempo que los primeros $15 \mathrm{~cm}$ de suelo, no obstante, en las 3 fechas de muestreo el $\mathrm{SMg}$ presentó la mayor acumulación en comparación al resto. Al comparar la presencia de $\mathrm{Mg}$ en las 2 profundidades se encontró a los 265 dds un incremento por arriba de $0,8 \mathrm{cmol}(+) .1^{-1}$ para los primeros $15 \mathrm{~cm}$ de suelo. Este resultado se presentó, debido a que el $\mathrm{SMg}$ es sulfato de magnesio con un contenido de 17,9\% de $\mathrm{MgO}$. La prueba de Simag también mostró aumentó en el nivel de Mg a través del tiempo, debido a que es una fuente de silicato de magnesio.

Las partes de $\mathrm{Si}$ en el suelo disminuyeron con el tiempo, con excepción del SKH que más bien aumentó (Cuadro 9). El SKH a los 175 dds presentó significativamente la mayor concentración de $\mathrm{Si}$ en el suelo (217,2 mg. $\left.\mathrm{l}^{-1}\right)$, este mismo comportamiento se encontró a los 265 dds y fue estadísticamente significativo al compararlo con las demás pruebas. El que mostró el menor contenido de silicio fue el Llane y difiere estadísticamente de las demás fuentes incluyendo el testigo. Cuando se evaluó la concentración de $\mathrm{Si}$ a una profundidad de $15-30 \mathrm{~cm}$, no se encontró diferencias entre las pruebas en los días 85 y 175 dds. Sin embargo, a los 265 dds el SKH mostró la mayor aglutinación de $\mathrm{Si}$, difiriendo estadísticamente de test, Llane y Simag. Al evaluar las 2 profundidades se encontró mayor tendencia de concentración de $\mathrm{Si}$ a 15 - 30 cm en comparación a los primeros $15 \mathrm{~cm}$ conforme pasa el tiempo. 


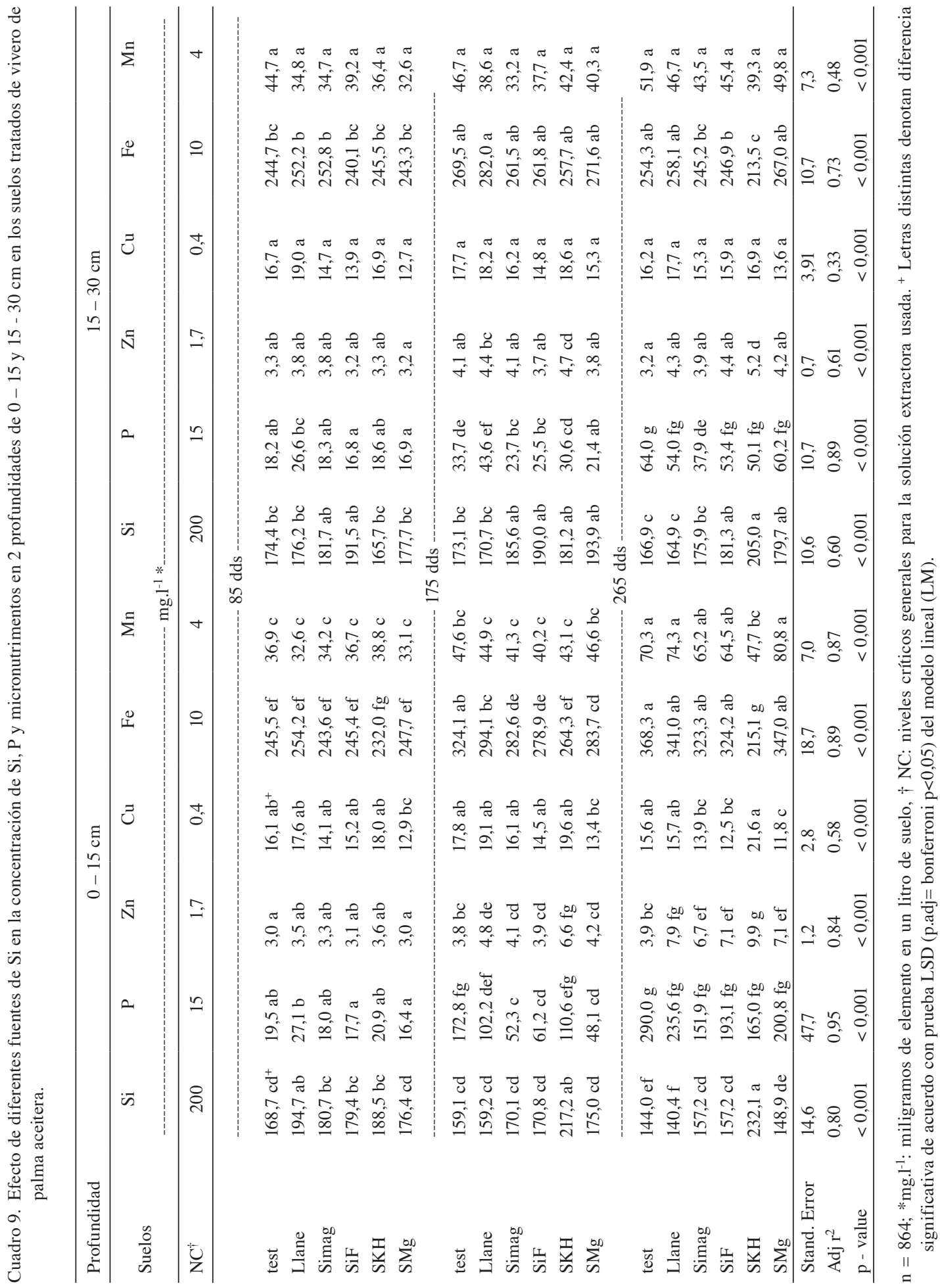


El P aumentó en el suelo a través del tiempo con mayor acumulación en la capa superficial del suelo que a más profundidad. La concentración de Zn en los suelos de vivero presentó incrementos en el tiempo para todos los tratamientos y fueron superiores al nivel crítico $\left(1,7 \mathrm{mg} . \mathrm{l}^{-1}\right)$ evaluados para inceptisoles (Cuadro 9). Al evaluar la concentración de $\mathrm{Zn}$ en los primeros 15 $\mathrm{cm}$ de profundidad se encontró que a los 85 dds ninguno de los tratamientos evidenció diferencias significativas $(\alpha=0,05)$, sin embargo, a los 175 y 265 dds el SKH presentó la mayor concentración de Zn (6,6 y 9,9 mg. l $\left.^{-1}\right)$ y difirió estadísticamente de los demás tratamientos. Las concentraciones de $\mathrm{Zn}$ a una profundidad de 15 - $30 \mathrm{~cm}$ mostraron diferencias en el SKH al compararlo con los demás tratamientos a los 175 y 265 dds. $\mathrm{Al}$ comparar ambas profundidades, presentaron tendencias de mayor acumulación de $\mathrm{Zn}$ a profundidades de $15-30 \mathrm{~cm}$ que a $0-15$ a los 85 dds, sin embargo, a los 265 este comportamiento fue inverso.

La concentración de $\mathrm{Cu}$, Fe y $\mathrm{Mn}$ no presentó cambios importantes en las primeras 2 fechas de evaluación en las 2 profundidades (Cuadro 9). El Cu reveló rangos de suficiencia ( $>0,4$ mg..$^{-1}$ ) en todos los tratamientos evaluados y en sus 2 profundidades, donde no se encontraron diferencias significativas $(\alpha<0,05)$. En relación con el Fe presentó rangos muy altos $\left(>232 \mathrm{mg} . \mathrm{l}^{-1}\right)$ en todos los tratamientos y manifestó diferencias entre las fechas de evaluación (85 - 265 dds) para los primeros $15 \mathrm{~cm}$ de profundidad, pero no significativas para profundidades entre $15-30 \mathrm{~cm}$. El SKH a los 265 dds dio la menor concentración significativamente del Fe en ambas profundidades. El Mn evidenció rangos de suficiencia en los suelos, estos estuvieron por arriba de 32 $\mathrm{mg} . \mathrm{l}^{-1}$. Este presentó un incremento significativo a los 265 dds, cuando se evaluó a profundidad de $0-15 \mathrm{~cm}$ y el SKH fue el que evidenció diferencias con la menor concentración de Mn en relación con los demás. El Mn evaluado a profundidad de $15-30 \mathrm{~cm}$ no manifestó diferencias estadísticas entre los tratamientos y las fechas evaluadas.

\section{Efecto de las fuentes de Si en la concentración de nutrimentos en las plantas de vivero}

El contenido de N, P, K, Ca, Mg, S y Si aumentó en los tratamientos que presentaron un crecimiento mayor en las plantas (Cuadro 10). El abc del contenido de $\mathrm{N}$ y $\mathrm{P}$, mostraron valores más altos $(224,2$ y 21,0$)$ en el Simag y difieren estadísticamente $(\alpha=0,05)$ de Llane y SKH. El K y Ca presentaron el mayor contenido en el Simag con diferencias al compararlos con SMg, SKH y Llane. En relación con el $\mathrm{Mg}$ y S presentó mayor contenido en el Simag y difieren numéricamente de Llane y SKH. El Si arrojó mayor contenido en Simag, test, $\mathrm{SMg}$ y $\mathrm{SiF}$ y presentó diferencias significativas con SKH y Llane. 
Cuadro 10. Efecto de las fuentes de Si en el índice de área bajo la curva en la concentración de nutrimentos en plantas de palma aceitera en vivero.

\begin{tabular}{|c|c|c|c|c|c|c|c|}
\hline \multirow{2}{*}{ Tratamientos } & $\mathrm{N}$ & $\mathrm{P}$ & $\mathrm{K}$ & $\mathrm{Ca}$ & $\mathrm{Mg}$ & S & $\mathrm{Si}$ \\
\hline & \multicolumn{7}{|c|}{ 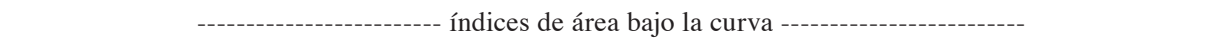 } \\
\hline Llane & $170,3 \mathrm{~b}^{+}$ & $17,1 \mathrm{~b}$ & $130,6 \mathrm{~b}$ & $72,2 \mathrm{c}$ & $24,6 \mathrm{bc}$ & $18,5 \mathrm{~b}$ & $78,4 \mathrm{c}$ \\
\hline $\mathrm{SMg}$ & $194,8 \mathrm{ab}$ & $16,7 \mathrm{~b}$ & $122,9 \mathrm{~b}$ & $72,4 \mathrm{bc}$ & $28,5 \mathrm{ab}$ & $18,8 \mathrm{~b}$ & $103,6 \mathrm{ab}$ \\
\hline Simag & $224,2 \mathrm{a}$ & $21,0 \mathrm{a}$ & $157,0 \mathrm{a}$ & $93,4 \mathrm{a}$ & $30,6 \mathrm{a}$ & $22,8 \mathrm{a}$ & $113,1 \mathrm{a}$ \\
\hline $\mathrm{SiF}$ & $194,6 \mathrm{ab}$ & $17,9 a b$ & $131,7 \mathrm{ab}$ & $82,8 \mathrm{abc}$ & $25,5 \mathrm{abc}$ & $20,2 \mathrm{ab}$ & $95,1 \mathrm{abc}$ \\
\hline SKH & $173,7 \mathrm{~b}$ & $16,4 \mathrm{~b}$ & $127,8 \mathrm{~b}$ & $67,9 \mathrm{c}$ & $22,9 \mathrm{c}$ & $17,9 \mathrm{~b}$ & $86,7 \mathrm{bc}$ \\
\hline test & $196,0 \mathrm{ab}$ & $18,8 \mathrm{ab}$ & $136,2 \mathrm{ab}$ & $86,8 \mathrm{ab}$ & $28,3 \mathrm{ab}$ & $21,0 \mathrm{ab}$ & $107,1 \mathrm{a}$ \\
\hline Stand. Error & 15,25 & 1,45 & 10,69 & 6,36 & 2,10 & 1,58 & 7,61 \\
\hline Adj r2 & 0,80 & 0,78 & 0,77 & 0,83 & 0,81 & 0,77 & 0,86 \\
\hline $\mathrm{p}$ - value & $<0,001$ & $<0,001$ & $<0,01$ & $<0,001$ & $<0,001$ & $<0,001$ & $<0,001$ \\
\hline \multirow{2}{*}{ Tratamientos } & $\mathrm{Al}$ & $\mathrm{Fe}$ & $\mathrm{Cu}$ & $\mathrm{Zn}$ & $\mathrm{Mn}$ & B & - \\
\hline & & (1) & . & bajo & va & 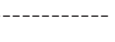 & \\
\hline Llane & 82936,5 b & 7394,2 b & $193,6 \mathrm{~b}$ & $251,1 \mathrm{bc}$ & $827,1 \mathrm{bc}$ & $184,3 \mathrm{~d}$ & - \\
\hline $\mathrm{SMg}$ & 99054,2 b & $9055,9 \mathrm{~b}$ & $208,6 \mathrm{ab}$ & $259,4 \mathrm{bc}$ & $858,7 \mathrm{bc}$ & $262,1 \mathrm{bc}$ & - \\
\hline Simag & 141069,2 a & $12548,3 \mathrm{a}$ & $267,5 \mathrm{a}$ & 333,1 a & $955,2 \mathrm{ab}$ & $286,5 \mathrm{ab}$ & - \\
\hline $\mathrm{SiF}$ & $105490,1 \mathrm{~b}$ & $9351,4 \mathrm{~b}$ & $251,4 \mathrm{ab}$ & $304,0 \mathrm{a}$ & $788,8 \mathrm{c}$ & $246,7 \mathrm{bc}$ & - \\
\hline SKH & $81002,1 \mathrm{~b}$ & $7260,4 \mathrm{~b}$ & $213,5 \mathrm{ab}$ & $245,7 \mathrm{c}$ & $613,1 \mathrm{~d}$ & $225,9 \mathrm{~cd}$ & - \\
\hline test & $76338,4 \mathrm{~b}$ & $6972,5 \mathrm{~b}$ & $208,8 \mathrm{ab}$ & $249,0 \mathrm{bc}$ & $1041,8 \mathrm{a}$ & $308,5 \mathrm{a}$ & - \\
\hline Stand. Error & 11920 & 990,3 & 24,0 & 23,1 & 66,3 & 18,8 & - \\
\hline Adj r2 & 0,87 & 0,88 & 0,75 & 0,83 & 0,90 & 0,90 & - \\
\hline $\mathrm{p}$ - value & $<0,001$ & $<0,001$ & $<0,001$ & $<0,001$ & $<0,001$ & $<0,001$ & - \\
\hline
\end{tabular}

$\mathrm{n}=2016 ;{ }^{+}$Letras distintas denotan diferencia significativa de acuerdo con prueba LSD (p.adj= bonferroni $\left.\mathrm{p}<0,01\right)$ del modelo lineal (LM).

Los contenidos de los nutrimentos $\mathrm{Al}, \mathrm{Fe}$, $\mathrm{Cu}, \mathrm{Zn}, \mathrm{Mn}$ y B presentaron mayores tendencias en las pruebas con mayor crecimiento. El Fe y Al mostraron la mayor concentración según el abc en Simag y difieren en $\alpha=0,05$ del resto de los tratamientos. El Cu evidenció la mayor abc $(267,5)$ en Simag y no presentó diferencias al resto de las pruebas a excepción del Llane, el cual presentó el menor contenido de $\mathrm{Cu}(193,6)$. El contenido de Zn fue mayor en Simag y SiF (333,1 y 304), estos difieren estadísticamente de los demás experimentos. El Mn y B revelaron el mayor contenido en el testigo y no difieren cuantitativamente del
Simag, los demás ensayos evidenciaron la menor concentración en estos micronutrimentos.

La concentración de N, P y K en la parte aérea y hoja 1 , mostró que el $\mathrm{N}$ tuvo tendencias de mayor concentración en la hoja 1 al compararlo con la parte aérea (Figura 1). El P presentó una menor concentración en la parte aérea en el testigo. El K mayores tendencias de concentración en la hoja 1 a excepción del Llane, que evidenció una concentración similar. Asimismo, el Ca mostró mayor concentración en la parte aérea. Los contenidos de $\mathrm{Mg}$ presentaron un comportamiento similar al $\mathrm{Ca}$, debido a que reveló mayor 
concentración en la parte aérea y fue similar en la hoja 1 en el SMg. El Si presentó mayor tendencia a acumularse en la parte aérea que en la hoja 1, al observar detalladamente el test, SiF y Simag tendieron a concentrar mucho más Si en la parte aérea que los demás tratamientos.

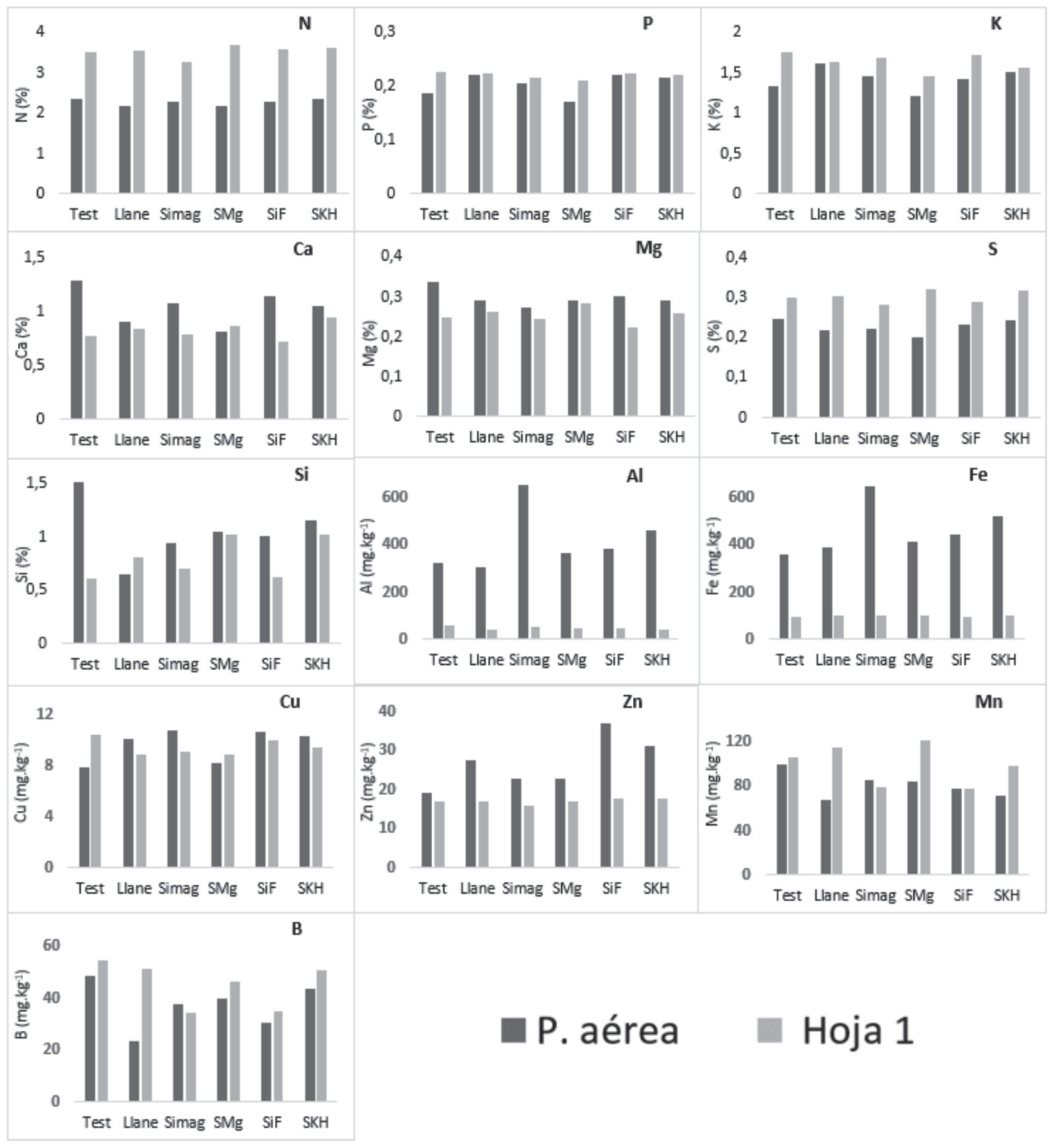

Fig. 1. Concentración de nutrimentos en la parte aérea de la planta y hoja número 1 a los 300 dds, tratadas con distintas fuentes de Si en mezcla al suelo en fase de vivero. 
Las concentraciones de $\mathrm{Al}$ y Fe mostraron diferencias importantes en la parte aérea con referencia a la concentración de la hoja 1 , puesto que el Simag es el que mostró mayor tendencia de acumulación en ambos nutrimentos. El $\mathrm{Cu}$ presentó concentraciones variables según el tratamiento, en relación con el test y $\mathrm{SMg}$ manifestaron tendencias en la hoja 1 que la parte aérea, mientras que en las demás pruebas el comportamiento fue inverso. El $\mathrm{Zn}$ evidenció mayor concentración en la parte aérea y es importante destacar que el SiF mostró la mayor tendencia al compararlos con los demás. Al evaluar el Mn y B mostraron tendencias de mayor concentración en la hoja 1, sin embargo, el Simag y SiF presentaron para ambas estructuras tendencias muy similares en ambos nutrimentos.

Al evaluar la absorción de nutrimentos en las plantas, a los 300 dds se encontraron contenidos muy similares en $\mathrm{N}, \mathrm{P}, \mathrm{K}, \mathrm{Ca}, \mathrm{S}$ y $\mathrm{Si}$, mientras que en micronutrimentos se encontraron diferencias (Cuadro 11). Los nutrimentos N, P, K y S no mostraron diferencias significativas, mientras que Ca presentó diferencias entre Llane y $\mathrm{SiF}$ y testigo. El Si expuso la menor acumulación significativa en la prueba Llane cuando se comparó con el resto. Entre los micronutrimentos solo el Zn dio diferencias minimamente significativas en el $\mathrm{SiF}$ en comparación al resto de los ensayos, el cual presentó la mayor absorción.

Cuadro 11. Efecto de las fuentes de Si en los contenidos de nutrimentos a los 300 dds en plantas de palma aceitera en vivero.

\begin{tabular}{|c|c|c|c|c|c|c|c|}
\hline \multirow{2}{*}{ Tratamientos } & $\mathrm{N}$ & $\mathrm{P}$ & $\mathrm{K}$ & $\mathrm{Ca}$ & $\mathrm{Mg}$ & S & $\mathrm{Si}$ \\
\hline & \multicolumn{7}{|c|}{ 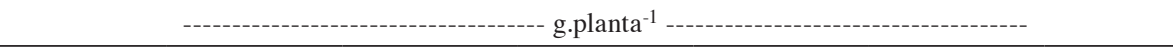 } \\
\hline Llane & $2,68 \mathrm{a}^{+}$ & $0,27 \mathrm{a}$ & $2,16 \mathrm{a}$ & $1,07 \mathrm{~b}$ & $0,37 \mathrm{ab}$ & $0,30 \mathrm{a}$ & $0,85 \mathrm{c}$ \\
\hline $\mathrm{SMg}$ & $3,15 \mathrm{a}$ & $0,25 \mathrm{a}$ & $1,96 \mathrm{a}$ & $1,14 \mathrm{ab}$ & $0,45 \mathrm{a}$ & $0,32 \mathrm{a}$ & $1,61 \mathrm{ab}$ \\
\hline Simag & $3,04 \mathrm{a}$ & $0,28 \mathrm{a}$ & $2,18 \mathrm{a}$ & $1,40 a b$ & $0,41 \mathrm{ab}$ & $0,33 \mathrm{a}$ & $1,57 \mathrm{ab}$ \\
\hline $\mathrm{SiF}$ & $2,95 \mathrm{a}$ & $0,29 \mathrm{a}$ & $2,08 \mathrm{a}$ & $1,43 \mathrm{a}$ & $0,41 \mathrm{ab}$ & $0,34 \mathrm{a}$ & $1,57 \mathrm{ab}$ \\
\hline SKH & $2,38 \mathrm{a}$ & $0,22 \mathrm{a}$ & $1,65 \mathrm{a}$ & $1,05 \mathrm{~b}$ & $0,31 \mathrm{~b}$ & $0,27 \mathrm{a}$ & $1,31 \mathrm{~b}$ \\
\hline test & $2,84 \mathrm{a}$ & $0,24 \mathrm{a}$ & $1,85 \mathrm{a}$ & $1,49 \mathrm{a}$ & $0,42 \mathrm{a}$ & $0,33 \mathrm{a}$ & $1,87 \mathrm{a}$ \\
\hline Stand. Error & 0,32 & 0,03 & 0,23 & 0,15 & 0,04 & 0,04 & 0,16 \\
\hline Adj r2 & 0,57 & 0,56 & 0,56 & 0,74 & 0,65 & 0,49 & 0,88 \\
\hline $\mathrm{p}$ - value & 0,06 & 0,05 & 0,07 & $<0,001$ & $<0,02$ & 0,16 & $<0,01$ \\
\hline Tratamientos & $\mathrm{Al}$ & $\mathrm{Fe}$ & $\mathrm{Cu}$ & $\mathrm{Zn}$ & Mn & B & - \\
\hline Llane & $670,3 \mathrm{e}$ & $72,6 \mathrm{~d}$ & $2,50 \mathrm{~b}$ & $3,81 \mathrm{~b}$ & $8,30 \mathrm{~b}$ & $3,13 \mathrm{c}$ & - \\
\hline $\mathrm{SMg}$ & $1302,5 \mathrm{bc}$ & $121,2 \mathrm{bc}$ & $3,22 \mathrm{ab}$ & $3,93 \mathrm{~b}$ & $12,4 \mathrm{a}$ & $5,85 \mathrm{ab}$ & - \\
\hline Simag & 2299,2 a & 205,4 a & $3,11 \mathrm{ab}$ & $3,80 \mathrm{~b}$ & $13,0 \mathrm{a}$ & $5,62 \mathrm{ab}$ & - \\
\hline $\mathrm{SiF}$ & $1570,3 \mathrm{~b}$ & $146,8 \mathrm{~b}$ & $4,11 \mathrm{a}$ & $5,70 \mathrm{a}$ & $11,2 \mathrm{a}$ & $4,62 \mathrm{~b}$ & - \\
\hline SKH & $1105,9 \mathrm{~cd}$ & $106,1 \mathrm{~cd}$ & $3,21 \mathrm{ab}$ & $3,67 \mathrm{~b}$ & $8,14 \mathrm{~b}$ & $4,89 \mathrm{ab}$ & - \\
\hline test & 818,2 de & $82,7 \mathrm{~d}$ & $2,51 \mathrm{~b}$ & $2,76 \mathrm{~b}$ & $12,2 \mathrm{a}$ & $6,02 \mathrm{a}$ & - \\
\hline Stand. Error & 160,4 & 14,7 & 0,43 & 0,50 & 1,16 & 0,53 & - \\
\hline Adj r2 & 0,95 & 0,94 & 0,72 & 0,84 & 0,83 & 0,86 & - \\
\hline $\mathrm{p}$ - value & $<0,01$ & $<0,01$ & $<0,01$ & $<0,01$ & $<0,01$ & $<0,01$ & - \\
\hline
\end{tabular}

$\mathrm{n}=312 ;{ }^{+}$Letras distintas denotan diferencia significativa de acuerdo con prueba LSD (p.adj= bonferroni $\left.\mathrm{p}<0,01\right)$ del modelo lineal (LM). 
La eficiencia de fertilización (EF) en los viveros de palma aceitera generalmente fue muy baja cuando se evaluó a los 300 dds y esta varía según la fuente de silicio y calcio que se aplicó en mezcla al suelo (Cuadro 12). En $\mathrm{N}$ tuvo la mayor EF en el SMg (23\%) en comparación al resto de los tratamientos, la EF más baja registrada se observó en el test (12,3\%). El P expresado en $\mathrm{P}_{2} \mathrm{O}_{5}$ reflejó la mayor $\mathrm{EF}$ en el $\mathrm{SiF}(16,5 \%)$, mientras que el testigo presentó la menor $(2,35 \%)$. El K representado en $\mathrm{K}_{2} \mathrm{O}$ reveló la mayor $\mathrm{EF}$ en el Simag y reveló la menor EF en el test.

Cuadro 12. Comparación de eficiencia de fertilización en nutrimentos aplicados en tratamientos con distintas fuentes de Si en mezcla al suelo en vivero de palma aceitera.

\begin{tabular}{|c|c|c|c|c|c|c|c|c|c|}
\hline Tratamiento & Rubro & $\mathrm{N}$ & $\mathrm{P}_{2} \mathrm{O}_{5}$ & $\mathrm{~K}_{2} \mathrm{O}$ & $\mathrm{CaO}$ & $\mathrm{MgO}$ & $\mathrm{SO}_{4}$ & $\mathrm{SiO}_{2}$ & B \\
\hline \multirow{3}{*}{ Llane } & Requisito (g.planta ${ }^{-1}$ ) & 2,7 & 0,6 & 2,6 & 1,5 & 0,6 & 0,9 & 1,8 & 0,01 \\
\hline & Dosis aplicada (g.planta ${ }^{-1}$ ) & 13,7 & 4,1 & 11,4 & 32,1 & 2,1 & 9,1 & 177,0 & 0,26 \\
\hline & $\%$ eficiencia & 19,6 & 15,2 & 22,8 & 4,7 & 28,8 & 9,9 & 1,0 & 3,90 \\
\hline \multirow{3}{*}{$\mathrm{SMg}$} & Requisito (g.planta ${ }^{-1}$ ) & 3,2 & 0,6 & 2,4 & 1,6 & 0,7 & 1,0 & 3,5 & 0,02 \\
\hline & Dosis aplicada (g.planta ${ }^{-1}$ ) & 13,7 & 4,1 & 11,1 & 4,3 & 2,1 & 21,2 & 30,0 & 0,26 \\
\hline & $\%$ eficiencia & 23,0 & 14,1 & 21,2 & 37,6 & 34,6 & 4,7 & 11,5 & 7,20 \\
\hline \multirow{3}{*}{ Simag } & Requisito (g.planta-1) & 3,0 & 0,6 & 2,6 & 2,0 & 0,7 & 1,0 & 3,4 & 0,02 \\
\hline & Dosis aplicada (g.planta ${ }^{-1}$ ) & 13,7 & 4,1 & 11,1 & 3,7 & 2,1 & 4,7 & 30,0 & 0,26 \\
\hline & $\%$ eficiencia & 22,2 & 15,7 & 23,5 & 52,8 & 31,4 & 21,3 & 11,2 & 7,00 \\
\hline \multirow{3}{*}{$\mathrm{SiF}$} & Requisito (g.planta ${ }^{-1}$ ) & 3,0 & 0,7 & 2,5 & 2,0 & 0,7 & 1,0 & 3,4 & 0,01 \\
\hline & Dosis aplicada (g.planta ${ }^{-1}$ ) & 13,7 & 4,1 & 11,2 & 7,4 & 2,1 & 7,1 & 30,0 & 0,26 \\
\hline & $\%$ eficiencia & 21,5 & 16,5 & 22,3 & 27,3 & 32,0 & 14,1 & 11,2 & 5,70 \\
\hline \multirow{3}{*}{ SKH } & Requisito (g.planta ${ }^{-1}$ ) & 2,4 & 0,5 & 2,0 & 1,5 & 0,5 & 0,8 & 2,8 & 0,02 \\
\hline & Dosis aplicada (g.planta ${ }^{-1}$ ) & 13,7 & 4,1 & 38,1 & 0,01 & 2,1 & 2,8 & 30,0 & 0,26 \\
\hline & $\%$ eficiencia & 17,4 & 12,3 & 5,2 & $>200$ & 24,0 & 29,3 & 9,4 & 6,10 \\
\hline \multirow{3}{*}{ test } & Requisito (g.planta ${ }^{-1}$ ) & 2,8 & 0,5 & 2,2 & 2,0 & 0,7 & 1,0 & 4,0 & 0,02 \\
\hline & Dosis aplicada (g.planta ${ }^{-1}$ ) & 23,0 & 23,0 & 50,0 & 0,01 & 14,0 & 14,0 & 0,01 & 0,52 \\
\hline & $\%$ eficiencia & 12,3 & 2,4 & 4,4 & $>200$ & 4,8 & 7,0 & $>200$ & 3,70 \\
\hline
\end{tabular}

$\mathrm{n}=312 ;{ }^{+}$Letras distintas denotan diferencia significativa de acuerdo con prueba LSD (p.adj= bonferroni $\left.\mathrm{p}<0,01\right)$ del modelo lineal (LM).

En relación con el Ca, el cual fue suministrado por las fuentes de $\mathrm{Si}$, evidenció que el testigo y SKH presentaron la mayor EF, ya que estos tratamientos no aportaron $\mathrm{Ca}$, sin embargo, al comparar los tratamientos que fueron aplicados con $\mathrm{Ca}$, mostraron que el Simag presentó la mayor EF y que el Llane mostró la menor EF. El $\mathrm{Mg}$, representado en $\mathrm{MgO}$, manifestó que el $\mathrm{SMg}$ presentó la mayor eficiencia $(34,6)$ y el testigo la menor (4,8\%). El S expresado en $\mathrm{SO}_{4}$ reveló que el $\mathrm{SiF}$ evidenció la mayor $(36,4 \%)$ EF y que el test la menor $(7,02 \%)$.

El Si en términos de $\mathrm{SiO}_{2}$ demostró que el SMg obtuvo la mayor EF $(11,5 \%)$ entre los ensayos que se aplicó silicio y el Llane fue el que evidenció la menor EF $(1,0 \%)$ en comparación al resto. Es importante destacar que el test presentó la mayor EF (>200\%) cuando se compararon 
todas las pruebas. Por último, el $\mathrm{B}$ mostró que el SMg presentó la mayor EF (7,20\%) y el test la menor $(3,70 \%)$.

\section{Análisis multivariado de acumulación de nutrimentos en las plantas y concentración de nutrimentos en el suelo en relación con la severidad}

Con el fin de encontrar una posible estructura con cambios en las variables químicas del suelo y las plantas en relación con la severidad, se realizó un análisis de componentes principales
(ACP). Dicho análisis mostró que las primeras 2 dimensiones, para los nutrimentos del suelo, explicaron un $69 \%$ y para los nutrimentos acumulados en las plantas, explicaron un $94 \%$ de la varianza observada en vivero (Figura 2). La primera dimensión que describe el comportamiento de los nutrimentos con la severidad, explicó un $50,1 \%$ de la variación del sistema y la segunda un $18,7 \%$. La primera dimensión, que describe la acumulación de esos componentes en la planta, explicó un 86,6\% de la variación y la segunda un 7,1\% según el mapa de factores de individuos. a

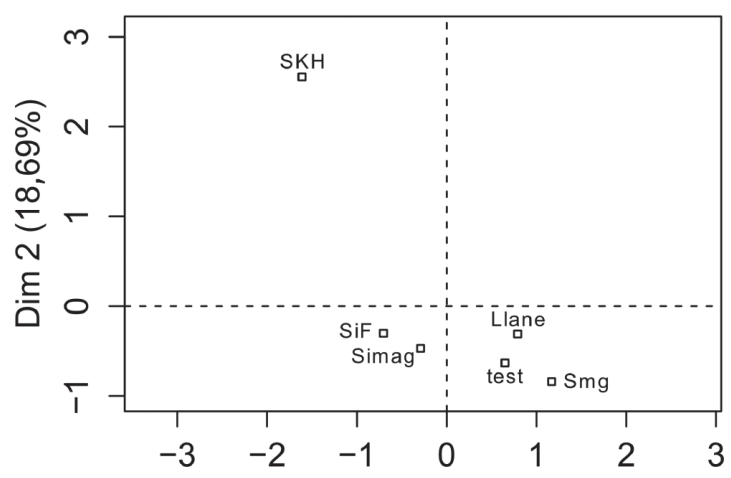

c $\quad \operatorname{Dim} 1(50,11 \%)$

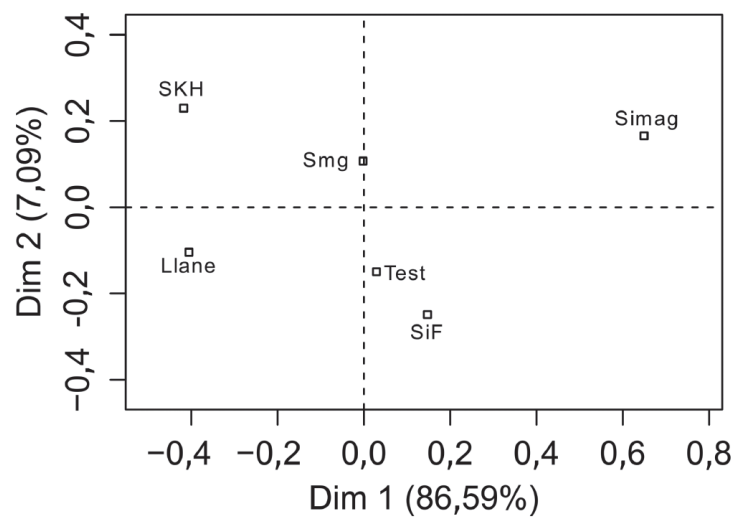

b
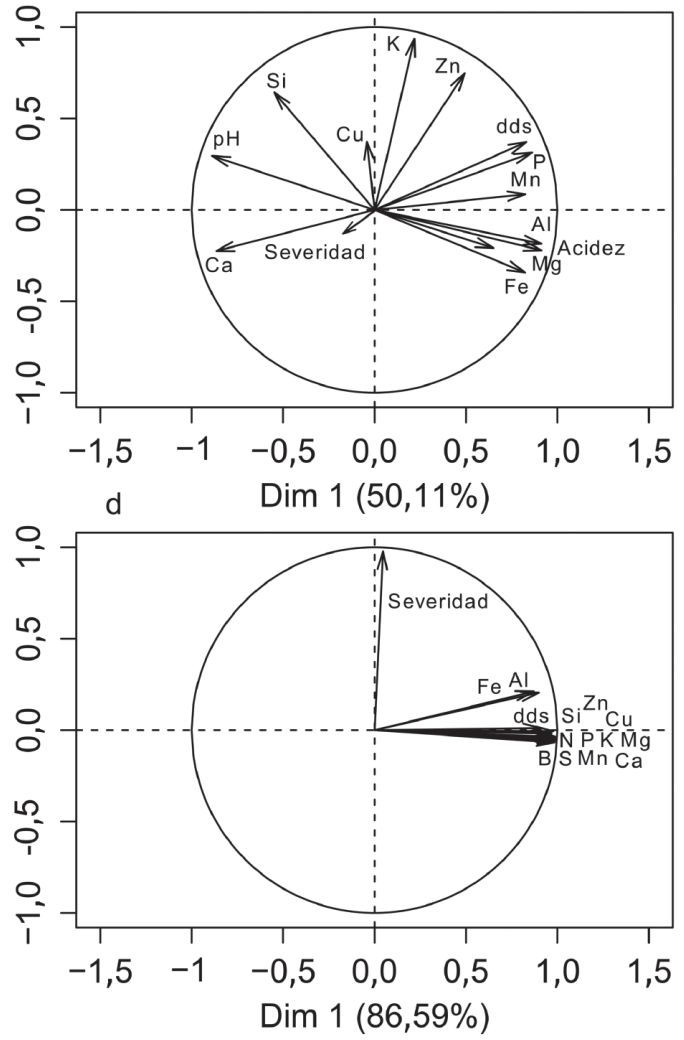

Fig. 2. Análisis de componentes principales para el contenido nutricional en el suelo, acumulación y severidad en palmas de vivero. a) Mapa de factor de individuos en dimensiones 1 y 2 para concentración de nutrimentos en suelos b) mapa de factor de variables en dimensiones 1 y 2 para concentración de nutrimentos en suelos. c) mapa de factor de individuos en dimensión 1 y 2 para contenido de nutrimentos en plantas. d) mapa de factor de variables en dimensiones 2 y 3 para contenidos de nutrimentos en plantas. 
El ACP agrupó los distintos tratamientos, según su similitud en las concentraciones de nutrimentos en el suelo, de acuerdo con la variación que presentó el suelo de las bolsas (Figura 2a, b). El ACP separó los distintos tratamientos en 3 grupos según la dimensión 1 y 2 , el primer grupo fue conformado por el $\mathrm{SKH}$, el segundo por $\mathrm{SiF}$ y Simag, el tercer grupo fue asignado para Llane, test y SMg. Estos grupos fueron seleccionados según una relación dimensional con respecto a las variables químicas de suelo $\mathrm{y}$, posiblemente, su relación en el comportamiento en las concentraciones obtenidas con el tiempo. Al observar el comportamiento dimensional de las variables químicas con la severidad y el tiempo (dds), se encontró que la severidad evidenció correlación directa con los contenidos de $\mathrm{Ca}$ en el suelo y expuso un comportamiento inverso a los de $\mathrm{Zn}, \mathrm{K}$, $\mathrm{P}, \mathrm{Mn}$ y los dds. Por otro lado, el Si presentó correlación directa con los contenidos de $\mathrm{Cu}$ y el pH de los suelos, además, corroboraron una correlación inversa con los niveles de acidez, Mg, Fe y Al.

$\mathrm{Al}$ observar el comportamiento de la acumulación de nutrientes en las plantas según el ACP, mostró una alta correlación con el tiempo, pero poco importante con la severidad (Figura 2c, d). El ACP encontró solo una agrupación con 2 tratamientos al ser los demás separados en distintas coordenadas según la dimensión 1 y 2 . El grupo fue conformado por el $\mathrm{SiF}$ y test al mostrar un comportamiento inverso con $\mathrm{SMg}$ y $\mathrm{SKH}$, según la relación dimensional con respecto a la acumulación de nutrimento en el tiempo, además, que no se encontró una relación con respecto a la severidad. Al parecer solo se encontró una tendencia baja de relación con el Fe y Al con la severidad, sin embargo, ambos nutrimentos tienen más relación con el resto de los nutrimentos, los cuales llevan una correlación más fuerte con el tiempo (dds).

\section{DISCUSIÓN}

Las plantas de vivero mostraron mayor tolerancia a enfermedades foliares en el $\mathrm{SiF}$ y un mayor crecimiento en el Simag en el tiempo (Cuadro 5). Es muy probable que este efecto sea producto de los desbalances de nutrimentos en el suelo aluvial, la reducción de la acidez intercambiable provocada por la sobre fertilización y la disponibilidad de micronutrimentos en la mejora de la nutrición de las plántulas en viveros de palma aceitera.

Al observar el pH desde los 85 dds se encontró que el $\mathrm{SiF}$ y Simag presentaron un $\mathrm{pH}$ por arriba de 5,0, mientras que los demás tratamientos disminuyeron a excepción de SKH, debido a que este último producto se suministraba cada 60 días. Este efecto se observó detalladamente en las concentraciones de acidez intercambiable $(1,16$ - 1,34 $\left.\operatorname{cmol}(+) . l^{-1}\right)$ y aluminio intercambiable $(0,79-0,93$ $\left.\mathrm{cmol}(+) . \mathrm{l}^{-1}\right)$, donde las cantidades fueron superiores al nivel crítico $\left(0,5 \mathrm{cmol}(+) \cdot \mathrm{l}^{-1}\right)$, producto de la acidificación de los suelos por los fertilizantes suministrados, lo cual se encuentra bien documentado (Molina 1998, Zapata 2004). Esta acidificación se respalda al observar las mayores concentraciones en los primeros $15 \mathrm{~cm}$ de profundidad, cuando se compararon con las de $15-30 \mathrm{~cm}$. Es probable que el efecto buffer sea producto de las cantidades de $\mathrm{CaO}$ y $\mathrm{MgO}$ que tiene Simag (3,72\%, $32,9 \%)$ y $\mathrm{SiF}(17,1 \%, 0,64 \%)$ respectivamente, los cuales actúan al realizar un efecto de corrección de acidez en el tiempo. El efecto de corrección de acidez está bien documentado en suelos ácidos (Espinosa y Molina 1999, Bernier y Alfaro 2006). Por otro lado, el Si también es usado en forma de enmienda para la corrección de los problemas de acidez, lo que es probable que también presentara un efecto en la reducción de esta en el suelo de las bolsas de vivero.

El Si mostró concentraciones por debajo del NC (200 mg. $\left.\mathrm{l}^{-1}\right)$ establecido para los suelos en Costa Rica (Lobo 2013), solo el SKH mantuvo sus contenidos por arriba de $\mathrm{NC}$, lo cual fue el único producto que aumentó el $\mathrm{Si}$ en los suelos. El contenido de $\mathrm{Si}$ en estos suelos rondaron entre

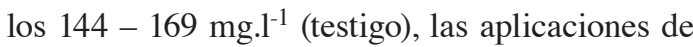
las fuentes de $\mathrm{Si}$ al inicio aumentaron significativamente, sin embargo, conforme fue pasando el tiempo esto disminuyó, ya que fue menor entre los primeros $15 \mathrm{~cm}$ de profundidad y mayor concentración entre $15-30 \mathrm{~cm}$. Esto también está 
relacionado inversamente con la acidez intercambiable causada por los fertilizantes (Lucas et al. 1993). La mayor presencia de Si a los 265 días en el suelo aportó la fuente de SKH. El Llane, a pesar de que suministró la mayor cantidad de Si (177 g $\mathrm{SiO}_{2}$ por bolsa) en comparación a los demás tratamientos, no mostró diferencias estadísticamente con el testigo. Al analizar el orden de solubilidad de silicio, según el método validado de $\mathrm{NA}_{2} \mathrm{CO}_{3}-$ $\mathrm{NH}_{4} \mathrm{NO}_{3}$ (Sebastian et al. 2013), se determinó que la fuente de serpentina (SMg) presentó mayor solubilidad de Si que la Zeolita (Llane) y las diatomitas (SiF) (Cuadro 1). Esto comprobó que las fuentes de Zeolitas no son adecuadas para los suelos usados en vivero de palma en Costa Rica (inceptisoles) y que las fuentes de silicio soluble (SKH), serpentinas y diatomitas mostraron una mejor solubilidad y efecto en la concentración química de los nutrimentos en el suelo a los 265 dds. Además, en Inceptisoles la concentración de Si es alta, debido a que estos suelos son de arcillas mixtas (Alvarado et al. 2014), los cuales tienen una liberación constante de $\mathrm{Si}$ producto de la sustitución isomorfa en las configuraciones laminares (Matichenkov et al. 2000, Zapata 2004, Datnoff et al. 2007), lo cual probablemente pudo ser acelerado por la acidificación inducida por los fertilizantes nitrogenados. Por otro lado, el $\mathrm{P}$ mostró una alta acumulación en los suelos de las bolsas producto de la fertilización que llevaba las fórmulas aplicadas en la fertilización de vivero.

Los aportes de nutrimentos dieron un efecto directo con el crecimiento de las plantas. El Simag mostró la mayor acumulación de nutrimentos, lo cual es determinado por la biomasa acumulada en el tiempo. El índice de área bajo la curva no determina la cantidad de nutrimentos (masa) acumulados, pero sí es sensible en determinar el efecto acumulativo entre 2 y más fechas mediante sus índices. El SiF no se diferencia significativamente del Simag en la acumulación de nutrimentos, pero al evaluar los contenidos de $\mathrm{Zn}$ y $\mathrm{Mn}$ en la parte foliar y parte aérea de la planta, se encontraron tendencias de una mayor concentración en los tejidos para el $\mathrm{Zn}, \mathrm{Cu}$ en el tratamiento en comparación al resto. Esto queda demostrado en el Cuadro 12, ya que determinó mayor acumulación a los 300 dds. Además, el Mn presentó una tendencia de estabilización en las concentraciones de la parte aérea y hoja 1 a los 300 dds.

La eficiencia de fertilización calculada para cada tratamiento mostró que las plantas en vivero son sobre abonados y el efecto de las altas concentraciones de los nutrimentos en las bolsas provienen de esas fuentes. Si bien las fertilizaciones en los tratamientos se basaron según la curva de absorción (Ramírez y Muñoz 2011), se esperaba un aumento en las eficiencias reflejadas en los cálculos de los requerimientos, debido a que los nutrimentos no presentan pérdidas por lixiviación, lavado, o escorrentía, lo cual ocasionaría pérdidas importantes en los suelos. A partir de ese análisis se identificó que la fertilización efectuada con las fórmulas convencionales en vivero (testigo) mostraron las menores eficiencias en los nutrimentos que fueron aplicados con los planes utilizados en vivero, lo cual probablemente ocasione mayor gravedad en los desbalances de los suelos.

El comportamiento de la severidad de $\mathrm{CNF}$ en las plantas de vivero presentó relaciones con las concentraciones de $\mathrm{Ca}, \mathrm{Zn}, \mathrm{Cu}, \mathrm{K}, \mathrm{P}$ y $\mathrm{Mn}$ en los suelos. Los mapas de los factores calculados para el suelo relacionaron con $\mathrm{SiF}$ y Simag el comportamiento de los nutrimentos y la severidad, debido a que los tratamientos mostraron mejores características de crecimiento (Simag) y tolerancia a las enfermedades en el tiempo ( $\mathrm{SiF}$ ). Mientras que el mapa de factores en la acumulación de nutrimentos relacionó estos parámetros con Simag, debido a la mayor acumulación de nutrimentos por el tratamiento. El ACP determinó que la concentración de $\mathrm{Cu}$ y $\mathrm{Zn}$ correlaciona inversamente con la tolerancia a enfermedades foliares, es por lo que a una mayor concentración en los suelos y en las plantas, la severidad disminuya. Este mismo efecto también se observó en las concentraciones de $\mathrm{K}, \mathrm{P}$ y $\mathrm{Mn}$.

El ACP relacionó un comportamiento proporcional de la severidad con las concentraciones de $\mathrm{Ca}$ en los suelos y relacionó las concentraciones de Si con el tratamiento SKH. Probablemente, 
el desbalance causado en los suelos por este nutrimento sea el factor relacionado directamente con la nutrición del vivero. Estas tendencias se observaron claramente al analizar la concentración de $\mathrm{Ca}$ en la hoja 1, lo que mostró que el tratamiento $\mathrm{SiF}$ presentó la menor concentración en comparación al resto de los tratamientos.

Las concentraciones foliares respaldan el ACP, debido a que los nutrimentos mostraron tendencias a mayores acumulaciones en el SiF cuando se compararon con el resto de los tratamientos. Esto también se respalda con la acumulación de nutrimentos a los 300 dds, donde el $\mathrm{Zn}$ se acumula en el tratamiento SIF. Los datos concuerdan con investigaciones en cereales, donde encontraron que mayores acumulaciones de $\mathrm{Zn}$ incentivan una mayor tolerancia a enfermedades, al mostrar correlaciones inversas entre los mayores contenidos de Zn y severidad en las plantas (Thongbai et al. 1993). Además, se ha demostrado que las aplicaciones de $\mathrm{Zn}$ en el suelo reducen significativamente la severidad de enfermedades (Graham y Webb 1991, Grewal et al. 1996) y que el efecto de $\mathrm{Zn}$ con E. guinessis, en palmas adultas reportan algunas hipótesis sobre la vinculación de $\mathrm{Cu}, \mathrm{Zn}$ y Fe en la afectación de PC (Chinchilla y Duran 1998), lo cual establece una posible relación importante con enfermedades del cultivo.

\section{CONCLUSIONES}

El $\mathrm{Si}$ en suelos aluviales no mostró un efecto directo en el control total de las enfermedades foliares en vivero de palma aceitera. Pero evidenció que el tratamiento SKH logró aumentar las concentraciones de Si por arriba del nivel crítico en suelos significativamente. Asimismo, la fuente de diatomitas ( $\mathrm{SiF}$ ) manifestó mayor tolerancia a enfermedades, debido a que la enmienda facilita la absorción de micronutrimentos ( $\mathrm{Zn})$, lo cual mejora la nutrición de la planta. En relación con el crecimiento, la fuente de serpentinas (Simag) presentó el mayor vigor y desarrollo en las palmas en vivero, pero no controló el problema de enfermedades foliares.

\section{AGRADECIMIENTOS}

Departamento de Investigación y Desarrollo, Compañía Palma Tica. Centro de Investigaciones Agronómicas y Carrera de Agronomía Sede de Guanacaste, Universidad de Costa Rica.

\section{LITERATURA CITADA}

Abdi, H; Williams, I. 2010. Principal component analysis. Wiley Interdiscip. Rev. Comput. Stat. 2(4):433-459.

Acosta, A; Ramírez, F; Albertazzi, H. 2007. El papel del silicio en el desempeño de palmas con flecha seca en una plantación comercial de palma aceitera en Quepos, Costa Rica. Revista Palmas 28(1):389-393.

Aguirre, C; Chávez, T; García, P; Raya, J. 2007. El silicio en los organismos vivos. Revista Interciencia 32 (8):504-509.

Ahmed, M; Asif, M; Goyal, A. 2012. Silicon the NonEssential Benedicial Plant Nutrient to enhanced drought tolerance in wheat. Crop Plant. University Rawalpindi - University of Alberta, Edmonton, Ab. Pakistan - Canada. 240 p.

Alvarado, A; Mata, R; Chinchilla, M. 2014. Arcillas identificadas en suelos de Costa Rica a nivel generalizado durante el período 1931-2012: I historia, metodología de análisis y mineralogía de arcillas en suelos derivados de cenizas volcánicas. Agronomía Costarricense 38(1):75-106.

Anderson, J. 2012. Efecto a través del tiempo del uso de fertilizantes silicatados para la reducción del mal de panamá (Fusarium oxysporum f. sp. cubense) en el cultivar Gros Michel (musa AAA). Tesis Lic. Turrialba, Costa Rica, Universidad de Costa Rica. 104 p.

Arauz, F. 2011. Fitopatología un enfoque agroecológico. 2 ed. San José, Costa Rica. Edit. UCR. 514 p.

ASD. 2010. Establishment and management of oil palm nurseries (en línea). Coto 47, Costa Rica. Consultado 19 abr. 2013. Disponible en http://www.asd-cr.com/ images/nurserie-guide.pdf

Bernier, R; Alfaro, M. 2006. Acidez de los suelos y efectos del encalado. Boletín $\mathrm{N}^{\circ}$. 151. Instituto de Investigaciones Agropecuarias. INIA. Osomo, Chile. $46 \mathrm{p}$.

Borda, O; Baron, F; Gómez, M. 2007. El silicio como elemento benéfico en avena forrajera (Avena sativa) respuestas fisiológicas de crecimiento y manejo. Agronomía Colombiana 25(2):273-279.

Cabalceta, G; Molina E. 2006. Niveles críticos de nutrimentos en suelos de Costa Rica utilizando la solución extractora Mehlich 3. Agronomía Costarricense 30(2):31-44. 
Chinchilla, C; Duran, N. 1998. Manejo de problemas fitosanitarios en palma de aceite. Una perspectiva agronómica. Revista Palmas 19:242-256.

Datnoff, L; Rodríguez, F; Seebold, K. 2007. Silicon and Plant Disease. In Datnoff, L; Elmer E; Huber, D. (eds.). Mineral Nutrition and Plant Disease. The American Phytopathological Society. USA. p. 233-246.

Epstein, E. 1999. Silicon. Annual Review of Plant Physiology and Plant Molecular Biology 50(1):641-664.

Espinosa, J; Molina, E. 1999. Acidez y encalados de los suelos. Ecuador. International Plant Nutrition Institute. $42 \mathrm{p}$.

Graham, D; Webb, M. 1991. Micronutrients and disease resistance and tolerance in plants, In Mortvedt, J; Cox, F; Shuman, L; Welch, R. (eds.). Micronutrients in Agriculture, 2nd ed., Soil Science Society of America, Inc. Madison, Wisconsin, USA. p. 329-370.

Grewal, H; Graham, R; Rengel, Z. 1996. Genotypic variation in zinc efficiency and resistance to crown rot disease (Fusarium graminearum Schw. Group 1) in wheat. Plant Soil 186(1):219-226.

Islam, A; Saha, C. 1969. Effects of silicon on the chemical composition of rice plants. Plant and Soil 30(3):446-458.

Jolliffe, I. 2005. Principal Component Analysis. In Everitt, B; Howell, D. (eds.). Encyclopedia of Statistics in Behavioral Science. John Wiley \& Sons, Ltd, Chichester, UK. 488 p.

Lobo, A. 2013. Validación de un protocolo para la determinación de silicio $(\mathrm{Si})$ en muestras de suelo y tejidos vegetales. Tesis Lic. San Pedro, Costa Rica. Universidad de Costa Rica. 95 p.

Lucas, Y; Luizao, F; Chauvel, A; Rouiller, J; Nahon, D. 1993. The relation between biological activity of the rainforest and mineral composition of the soils. Science 260(1):521-523.

Ma, J; Yamaji, N. 2008. Functions and transport of silicon in plants. Cellular and molecular life sciences 65(1):3049-3057. https://doi.org/10.1007/s00018008-7580-x

Marschner, P; Marschner, H. 2012. Mineral nutrition of higher plants. USA. Academic press ELSEVIER. 672 p.

Matichenkov, V; Bocharnikova, E; Calvert, D; Snyder, G. 2000. Comparison study of soil silicon status in sandy soils of south Florida. Soil Crop Sci. Florida 59(1):132-137.

Molina, E. 1998. Encalado para la corrección de a acidez del suelo. San José, Costa Rica. Asociación Costarricense de la Ciencia del Suelo. 45 p.

Ortiz, R; Fernández, O. 2000. Cultivo de palma aceitera. San José, Costa Rica. EUNED. 208 p.

Quero, E. 2008. La Biosilicificación proceso biológico fundamental en la productividad vegetal (en línea). Portal de silicio en los sistemas biológicos. México. Consultado 11 jul 2013. Disponible en http://loquequero. com/portal/index.php?option=com_content\&task=view \&id $=27 \&$ Itemid $=1$

Ramírez, F; Muñoz, F. 2011. Curva de nutrientes para la etapa de vivero de tres materiales de palma aceitera (Elaeis guineensis Jacq.) (en línea). Congreso ecuatoriano de la ciencia del suelo. Santo Domingo, Ecuador. Consultado 19 abr. 2013. Disponible en http://www.secsuelo.org/XIICongreso/ Simposios/Nutricion/Magistrales/2.\%20Floria\%20 Ramirez.\%20Curva\%20materiales\%20vivero.\%20 PALMATICA,\%20Costa\%20Rica.pdf

Raya, J; Aguirre, C. 2012. El papel del silicio en los organismos y ecosistemas. Conciencia tecnológica 43(1):42-46

Regil, J. 2014. Eficiencia del ácido silicio para favorecer la absorción de fosforo en plantas de palma africana variedad deli x Nigeria, en etapas de vivero. Tesis de Lic. Asunción, Guatemala. Universidad Rafael Landivaer. 57 p.

Rodríguez, R; Jiménez, E. 2013. Procedimiento para la evaluación de medidas de crecimiento de la hoja 1 y muestreo foliar en plantas de vivero. Boletín Mensual de la Unidad de Investigación de Palma Tica S.A. y Cukra Development Corporation. San José, Costa Rica. 8 p.

Sebastian, D; Hugh, R; Kinsey, C; Korndorfer, G; Pereira, H; Buck, G; Datnoff, L; Miranda, S; Provance-Browley, M. 2013. A 5-day method for determination of soluble silicon concentrations in nonliquid fertilizer materials using a sodium carbonate - ammonium nitrate extractant followed by visible spectroscopy with heteropoly blue analysis: Single - Laboratory Validation. Journal of AOAC international 96(2):251-259.

Serrano, R. 2003. Introducción al análisis de datos experimentales: tratamiento de datos en bioensayos. Castellon de la Plana, España, Universitat Jaume I. $189 \mathrm{p}$.

Thongbai, P; Hannam, R; Grahan, R; Webb, M. 1993 Interaction between zinc nutritional status of cereals and Rhizoctonia root severity. Plant an Soil 151(1):207-214.

Ulloa, M; Ramírez, F. 2011. Resultado del diagnóstico sobre la necrosis foliar del vivero Coto 49. Boletín Mensual de la Unidad de Investigación de Palma Tica S.A. y Cukra Development Corporation. 3 p.

Ulloa, M; Serrano, E. 2012. Prueba de sensibilidad in vitro de Colletotrichum gloeosporioides y Curvularia lunata, principales agentes causales del Complejo de Necrosis Foliar (CNF) a diferentes fungicidas. Boletín Mensual de la Unidad de Investigación de Palma Tica S.A. y Cukra Development Corporation. 9 p.

Zapata, R. 2004. La química de la acidez del suelo. Sociedad Colombiana de la ciencia del suelo. Medellin, Colombia. 207 p. 